\title{
Pharmacological inhibition of Ref- 1 enhances the therapeutic sensitivity of papillary thyroid carcinoma to vemurafenib
}

\author{
Linfei $\mathrm{Hu}^{1,7}$, Jun Zhang ${ }^{2,7}$, Mengran Tian ${ }^{1,7}$, Ning Kang ${ }^{1}$, Guangwei Xu' ${ }^{1}$, Jingtai Zhi ${ }^{3}$, Xianhui Ruan (DD ${ }^{1}$, Xiukun Hou ${ }^{1}$, Wei Zhang ${ }^{1}$,
} Jiaoyu $\mathrm{Yi}^{1}$, Weike $\mathrm{Ma}^{1}$, Luchen Chang ${ }^{4}$, Tao Tang ${ }^{1,5}$, Xiangqian Zheng $\mathbb{D}^{1 凶}{ }^{\circledR}$, Xi Wei $\mathbb{D}^{4 凶}$ and Ming Gao (D)

(c) The Author(s) 2022

The use of the BRAF inhibitor vemurafenib exhibits drug resistance in the treatment of thyroid cancer (TC), and finding more effective multitarget combination therapies may be an important solution. In the present study, we found strong correlations between Ref- 1 high expression and BRAF mutation, lymph node metastasis, and TNM stage. The oxidative stress environment induced by structural activation of BRAF upregulates the expression of Ref-1, which caused intrinsic resistance of PTC to vemurafenib. Combination inhibition of the Ref-1 redox function and BRAF could enhance the antitumor effects of vemurafenib, which was achieved by blocking the action of Ref- 1 on BRAF proteins. Furthermore, combination treatment could cause an overload of autophagic flux via excessive AMPK protein activation, causing cell senescence and cell death in vitro. And combined administration of Ref-1 and vemurafenib in vivo suppressed PTC cell growth and metastasis in a cell-based lung metastatic tumor model and xenogeneic subcutaneous tumor model. Collectively, our study provides evidence that Ref- 1 upregulation via constitutive activation of BRAF in PTC contributes to intrinsic resistance to vemurafenib. Combined treatment with a Ref- 1 redox inhibitor and a BRAF inhibitor could make PTC more sensitive to vemurafenib and enhance the antitumor effects of vemurafenib by further inhibiting the MAPK pathway and activating the excessive autophagy and related senescence process.

Cell Death and Disease (2022)13:124; https://doi.org/10.1038/s41419-022-04550-0

\section{INTRODUCTION}

Papillary thyroid cancer (PTC) is the most common pathological subtype of thyroid cancer (TC), and its BRAF mutation rate, especially the point mutation of valine at the 600 positions to glutamate, i.e., BRAFV600E, can reach $>80 \%$ in Asian populations, which creates a very favorable condition for targeted therapy in this population. Currently, the BRAF inhibitors (BRAFis) approved by the Food and Drug Administration mainly include sorafenib, regorafenib, vemurafenib, dabrafenib, and encorafenib [1]; the last three are mainly used to treat patients with advanced melanoma, which also harbors a high BRAF mutation rate, and among them, vemurafenib, a BRAF ${ }^{\mathrm{V} 600}$-specific small-molecule inhibitor, has significantly changed therapeutic prospects and been shown to quickly inhibit the growth of melanoma and control malignant tumor progression in most patients [2,3]. To date, the application of vemurafenib in advanced PTC is still in clinical trials, showing potential clinical application value [4-6]. Experiments in vitro have revealed poor vemurafenib treatment sensitivity and early resistance [7-9] in PTC cell lines. Under these circumstances, higher drug concentrations are required to achieve a satisfactory inhibitory effect, which tremendously increases the risk of drug toxicity to achieve the same expected effect in vivo. At present, multiple studies have reported the mechanism of drug resistance in tumors, including activity attenuation of immune cells [10], bypass of mitogen-activated protein kinase (MAPK) pathway activity [11], loss of negative feedback [12, 13], and increased antiapoptotic protein $\mathrm{Bcl} 2$ expression [14]. However, there is no final conclusion at present on what causes an inadequate therapeutic index in PTC, melanoma, or other cancers regarding the mechanism of resistance, and novel mechanisms and strategies for combination drugs still need to be exploited.

Redox factor-1 (Ref-1), also known as apurinic/apyrimidinic endonuclease 1 (APE1), is a highly conserved functional enzyme that has a redox function that regulates the activity of a variety of important transcription factors and has nucleic acid endonuclease activity, allowing Ref- 1 to function as a DNA repair enzyme. In the

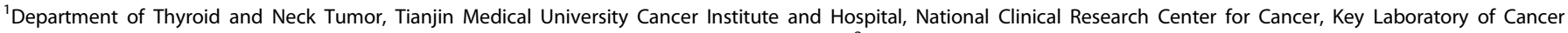
Prevention and Therapy, Tianjin's Clinical Research Center for Cancer, 300060 Tianjin, China. ${ }^{2}$ Department of Breast Cancer, Tianjin Medical University Cancer Institute and Hospital, Key Laboratory of Breast Cancer Prevention and Therapy, Key Laboratory of Cancer Prevention and Therapy, Tianjin's Clinical Research Center for Cancer, National

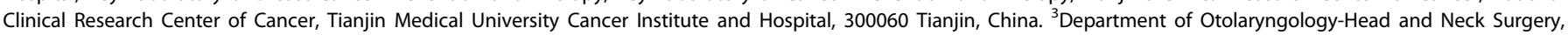

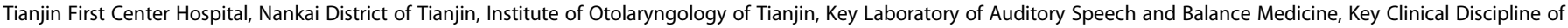

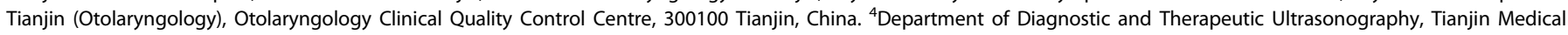
University Cancer Institute and Hospital, National Clinical Research Center for Cancer, Key Laboratory of Cancer Prevention and Therapy, Tianjin's Clinical Research Center for

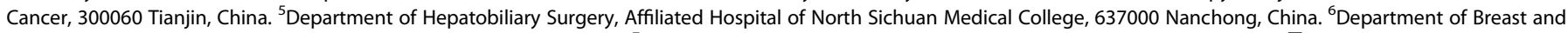

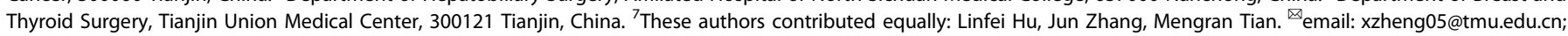
weixi@tmu.edu.cn; headandneck2008@126.com

Edited by Dr Giovanni Blandino
}

Received: 30 June 2021 Revised: 18 December 2021 Accepted: 13 January 2022

Published online: 08 February 2022 


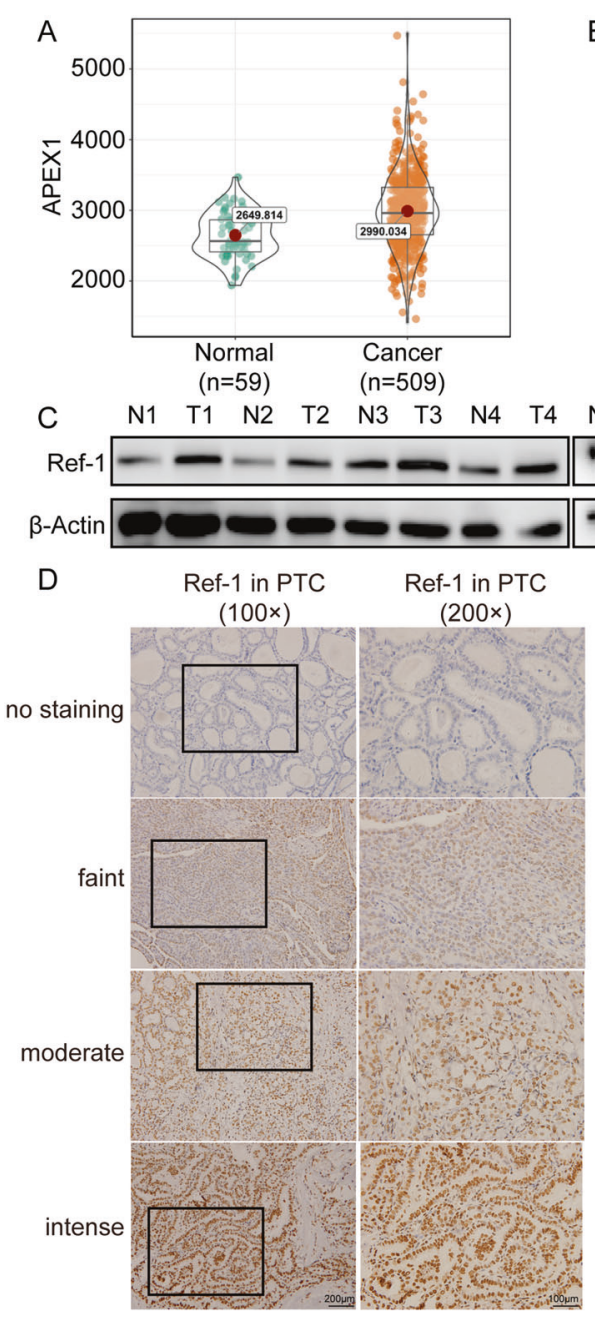

B

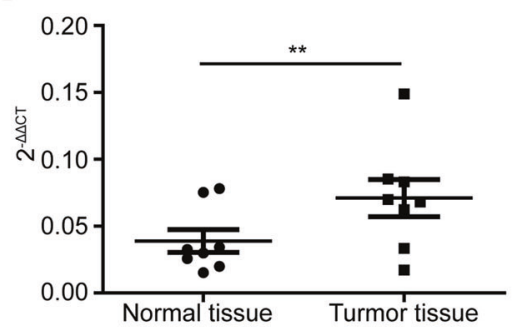

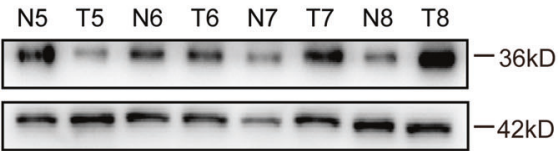

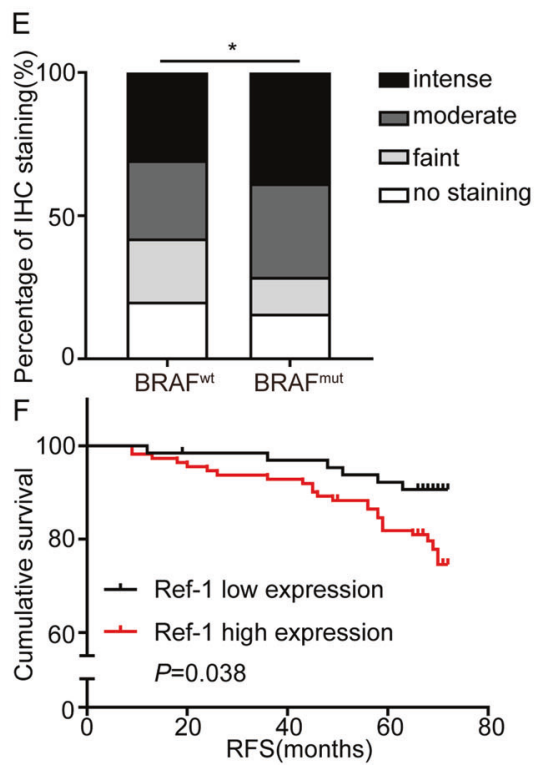

Fig. 1 Ref-1 expression was upregulated in PTC patient samples. A Amplification alteration of the APEX1 gene in thyroid cancer and normal tissue samples from The Cancer Genome Atlas (TCGA) database. B Real-time PCR detection of the mRNA expression level of Ref-1 in tumor and normal tissue samples from 16 patients. C Western blot detection of the protein expression level of Ref-1 in tumor and normal tissue specimens from 8 patients. D Representative immunohistochemical staining for Ref-1 in PTC specimens. E Percentages of samples with different BRAF mutation statuses and specific Ref- 1 expression levels among 178 PTC cases. F RFS analysis of groups based on the high and low expression of Ref- 1 among 178 PTC cases. ${ }^{*} P<0.05,{ }^{* *} P<0.01$.

past, numerous studies $[15,16]$ have verified that Ref- 1 is involved in the drug resistance process as a DNA repair enzyme in various tumors, and an increasing number of reports [17-19] have shown that Ref-1 can also regulate drug resistance through its redoxdependent activity. In a proteomic analysis, Salzano et al. [20] identified 13 differentially expressed proteins between differentiated rat thyroid cell line and derived undifferentiated cell line nuclear extracts, and Ref-1, one of the proteins that might be involved in a transcriptional mechanism, was suspected to be associated with the occurrence of TC. Liu et al. [21] indicated that the expression of Ref- 1 was decreased during the early stage of melanoma, while in later stages during the transformation into malignant metastatic melanoma with an increase in the expression level of redox activity of Ref- 1 . Another study revealed that upregulation of Ref-1 expression promoted melanoma resistance to BRAFis [22]. These previous reports raise the possibility that Ref1 may be an important causative factor in the differential response to drug therapy between PTC and melanoma.

In the present study, we found that Ref- 1 was highly expressed in TC and had a positive correlation with BRAF mutation and induced oxidation environment by MAPK pathway constitutive activation. A Ref-1 redox inhibitor could enhance the abilities of vemurafenib to inhibit proliferation and metastasis and promote apoptosis differentiation in vitro and in vivo. Additionally, the inhibitor could synergize with vemurafenib to cause cell senescence. Mechanistic studies of PTC cells in vitro demonstrated that Ref-1 reduced the antitumor effects of vemurafenib by binding to the BRAF protein and combined therapy with vemurafenib and E3330 caused cell senescence by overburdening autophagic flow.

\section{RESULTS}

Clinical Ref-1 expression was related to an inferior prognosis and BRAF mutation in PTC

To determine the expression of Ref- 1 in PTC, we first extracted sample data of 568 PTC patients (509 PTC samples and 59 normal samples) from The Cancer Genome Atlas (TCGA) database and then conducted quantitative real-time PCR (qPCR) and western blot analyses of normal and tumor fresh tissue samples from 16 PTC patients and 8 PTC patients, respectively. The analyses revealed that the mRNA and protein levels of Ref- 1 were both higher in tumor tissue than in normal tissue (Fig. 1A-C). To investigate the effects of Ref-1 expression levels on PTC patients, immunohistochemical (IHC) staining of 178 PTC samples, which were divided into four groups based on the Ref-1 staining score, was conducted (Fig. 1D). IHC 
Table 1. Analysis of Ref-1 expression and clinicopathological features in PTC.

\begin{tabular}{|llll}
\hline Vairables & Ref-1 expression & P value \\
\hline $\begin{array}{c}\text { Age, years } \\
<55\end{array}$ & 43 & $\begin{array}{l}\text { Moderate/ } \\
\text { high }\end{array}$ & \\
\hline$\geq 55$ & 22 & 68 & 0.428 \\
\hline Gender & & 45 & \\
\hline Male & 17 & & \\
\hline Female & 48 & 33 & 0.663 \\
\hline Multifocality & & 80 & \\
\hline No & 46 & & \\
\hline Yes & 19 & 79 & 0.904 \\
\hline BRAF mutation & & 34 & \\
\hline No & 40 & & \\
\hline Yes & 25 & 45 & 0.005 \\
\hline T stage & & 68 & \\
\hline T1 + T2 & 59 & & \\
\hline T3 + T4 & 6 & 101 & 0.767 \\
\hline LNM & & 12 & \\
\hline No & 38 & 103 & 0.296 \\
\hline Yes & 27 & 48 & 0.040 \\
\hline TNM stage & & 65 & \\
\hline I-II & 62 & & \\
\hline III-IV & 3 & 10 & \\
\hline
\end{tabular}

scoring and clinicopathological data analysis showed that high expression of Ref- 1 was positively correlated with BRAF mutation and lymph node metastasis (Table 1 and Fig. 1E) but not associated with patient sex, age, tumor diameter, number of lesions, T stage, or TNM stage (AJCC Eighth) and indicated that patients with negative Ref-1 staining had longer relapse-free survival than those with positive staining (Fig. 1F). PCR and western blot analysis of thyroid cell lines showed notably increased Ref-1 expression in BRAF ${ }^{\mathrm{V} 600 \mathrm{E}}$ cells (Supplemental Fig. 1A, B).

\section{An intrinsic high level of Ref-1 contributed to depressed vemurafenib sensitivity in BRAF ${ }^{\mathrm{V} 600 \mathrm{E}}$ PTC}

Then, sample data from 487 PTC patients (198 with BRAF ${ }^{\mathrm{wt}}$ and 289 with BRAF $^{\text {mut }}$ ) and 469 melanoma patients ( 229 with BRAF $^{\text {wt }}$ and 240 with BRAF $^{\text {mut }}$ ) extracted from the TCGA were divided into two groups according to the BRAF mutation status. Gene expression analysis revealed that BRAF mutation elevated the mRNA level of Ref- 1 in PTC $(P<0.0001)$, while melanoma exhibited the opposite pattern $(P=0.0115)$ (Fig. $2 A)$. The observation of positive correlations between the expression of Ref- 1 and markers of MAPK pathway activation in PTC but not in melanoma reconfirmed the patterns (Fig. 2B). BRAF mutation is closely related to intracellular oxidative stress, we analyzed the expression of ROS-producing enzyme NOX4 and its relationship with APEX1 in the TCGA database. The results showed that BRAF mutation was accompanied by high expression of NOX4, and the expression level of the latter is positively correlated with the expression of redox factor APEX1 (Fig. 2C, D). To further verify whether the increased expression of Ref- 1 is involved in BRAFi resistance in BRAF $^{\mathrm{V} 600 \mathrm{E}}$ PTC, we treated PTC cell lines (BCPAP and $\mathrm{K}-1$, both harboring the $\mathrm{BRAF}^{\mathrm{V} 600 \mathrm{E}}$ mutation) and a melanoma cell line (A375, harboring the BRAF ${ }^{\mathrm{V} 600 \mathrm{E}}$ mutation) with different concentrations of vemurafenib for IC50 testing, and the results showed a higher sensitivity in the melanoma cell line $\left(I C 50_{\mathrm{A} 375}=56.46 \mathrm{nM}\right)$ than in the PTC cell lines $\left(I C 50_{\mathrm{BCPAP}}=31.36 \mu \mathrm{M}, \quad \mathrm{IC} 50_{\mathrm{K}-1}=\right.$ $36.62 \mu \mathrm{M}$ ) (Fig. 2E). Next, we used different concentrations of E3330 combined with $10 \mu \mathrm{M}$ vemurafenib to treat TC cell lines BCPAP and K-1, and observed that vemurafenib can significantly increase the inhibitory effect of vemurafenib on cell proliferation at a concentration of $50 \mu \mathrm{M}$ (Fig. 2F). Then we treated BCPAP and K-1 cells with the Ref- 1 redox inhibitor E3330 for $24 \mathrm{~h}$ and then repeated the IC50 testing procedure for vemurafenib. To our surprise, pretreatment with E3330 distinctly increased the sensitivity of the PTC cell lines to vemurafenib (IC50 $50_{\text {BCPAP }}=$ $14.06 \mu \mathrm{M}, \quad \mathrm{IC} 50_{\mathrm{K}-1}=17.80 \mu \mathrm{M}$ ) (Fig. $2 \mathrm{G}$ ). Based on the above analysis, the expression level of Ref- 1 was increased in BRAF ${ }^{\mathrm{V} 600 \mathrm{E}}$ PTC with active redox systems, which indicates that Ref- 1 is involved in a mechanism for intrinsic resistance to a BRAFi in PTC.

\section{The Ref-1 redox inhibitor enhanced the sensitivity of BRAF $^{\text {V600E }}$ PTC to vemurafenib in vitro}

To assess the effect of vemurafenib and further evaluate the effect of combination treatment on PTC in vitro, BCPAP and K-1 cells, which harbor BRAF ${ }^{\mathrm{V} 600 \mathrm{E}}$, were exposed to vemurafenib or/and E3330 to detect the effects of the two inhibitors. As expected, CCK-8 and clone formation experiments revealed that E3330 could enhance the antitumor effect of vemurafenib (Fig. 3A-D). Using flow cytometry to detect BrdU, we found that the proportion of $S$-phase cells in the combination group was significantly reduced, which indicated that E3330 could enhance the antiproliferative effect of vemurafenib, and the results of cell cycle experiments suggested that proliferation was mainly arrested in the G1 phase (Fig. 3E-H). Next, apoptosis evaluation showed that E3330 could significantly enhance the proapoptotic property of vemurafenib (Fig. 4A, B), and this effect was mainly achieved via the mitochondrial apoptosis pathway (Fig. 4C, D), as determined by assessing Bcl2, Bax, cleaved-PARP (cl-PARP), cleaved-caspase3 (clcaspase3) and Survivin (Fig. 4E, F). Furthermore, transwell assay results showed that E3330 also enhanced the capacity of vemurafenib to inhibit migration and invasion (Supplemental Fig. 2A-D) and to affect E-cadherin and Vimentin levels in BCPAP and K-1 cell lines (Supplemental Fig. 2E, F). We analyzed the combined effects on angiogenesis and tumor differentiation in vivo and in vitro. The results show that both vemurafenib and E3330 could inhibit the angiogenesis of thyroid tumors and the combination treatment has a more significant inhibition effect in the angiogenesis phenotype (Supplemental Fig. 3A, B). In cell differentiation function analysis, both vemurafenib and E3330 can reduce the level of ROS in TC cells to a certain extent, and the combined treatment can further reduce the level of ROS, and the reduced ROS level can promote the differentiation of TC cells and induce cell differentiation (Supplemental Fig. 4A-C). The above results demonstrated that in combination therapy, E3330 played a role in sensitizing BRAF ${ }^{\mathrm{V} 600 \mathrm{E}}$ PTC cells to vemurafenib.

\section{Ref-1 regulated sensitivity to vemurafenib via a redox- dependent mechanism in PTC}

To further investigate the sensitizing mechanism of E3330, we detected the levels of the BRAF downstream proteins $p$-MEK and $p$-ERK in the classical MAPK pathway. Western blot analysis showed that the levels of phosphorylated MEK and ERK were significantly lower than those seen with vemurafenib treatment alone (Fig. 5A, B), so we speculated that Ref- 1 promotes or maintains the function of BRAF. Subsequently, we selected the BRAF wild-type cell line TPC-1 to construct a BRAF ${ }^{\mathrm{V} 600 \mathrm{E}}$-overexpressing stable cell line for Co-IP and the BCPAP cell line for small interfering RNA knockdown experiments to verify the mechanism linking BRAF and Ref-1. The results showed that Ref1 played a role in maintaining BRAF function by binding with BRAF (Fig. 5C, D). 

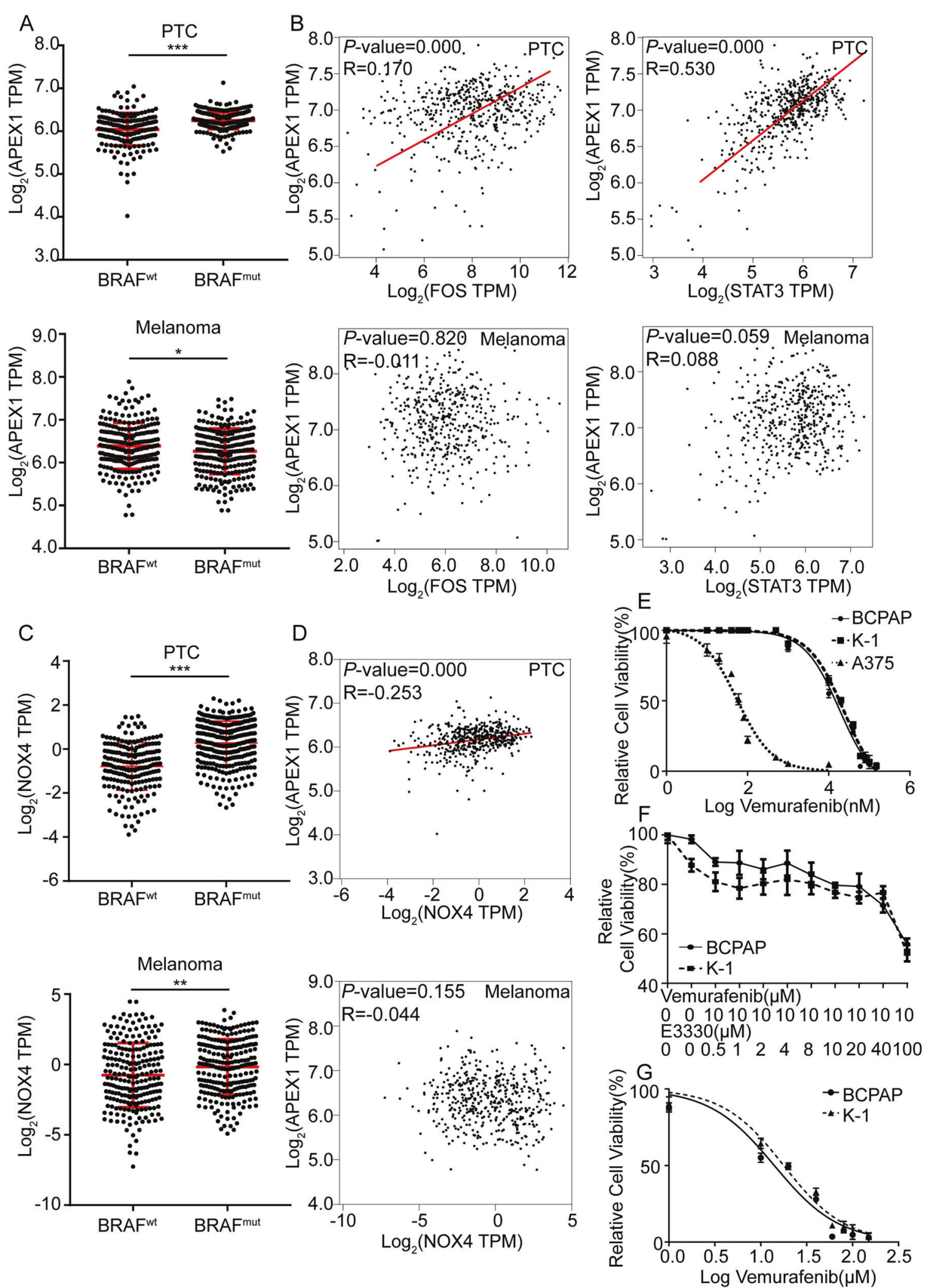

Fig. 2 Resistance to vemurafenib in PTC was related to upregulated Ref-1 expression. A Analysis of data from the TCGA database for Ref-1 mRNA expression levels in BRAF ${ }^{\mathrm{wt}}$ and BRAF ${ }^{\text {mut }}$ PTC (upper) and melanoma (bottom) cases. B Correlation analysis of Ref-1 and MAPK pathway targeting genes expression in PTC (upper) and melanoma (bottom) cases based on the TCGA database. C Analysis of data from the TCGA database for NOX4 mRNA expression levels in BRAF ${ }^{\mathrm{wt}}$ and BRAF ${ }^{\mathrm{mut}}$ PTC (upper) and melanoma (bottom) cases. D Correlation analysis of Ref-1 and NOX4 mRNA expression levels in PTC (upper) and melanoma (bottom) cases based on the TCGA database. E Vemurafenib IC 50 detection by a CCK-8 assay in BCPAP, K-1, and A375 cells. F Cell proliferation ability detection by a CCK-8 assay after vemurafenib (10 $\mu$ M) and E3330 (different concentration gradient) pretreatment in BCPAP and K-1 cells. G Vemurafenib $\mathrm{IC}_{50}$ detection after E3330 pretreatment by a CCK-8 assay in BCPAP and K-1 cells. ${ }^{*} P<0.05,{ }^{* *} P<0.01$. ${ }^{* *} P<0.001$. 
A

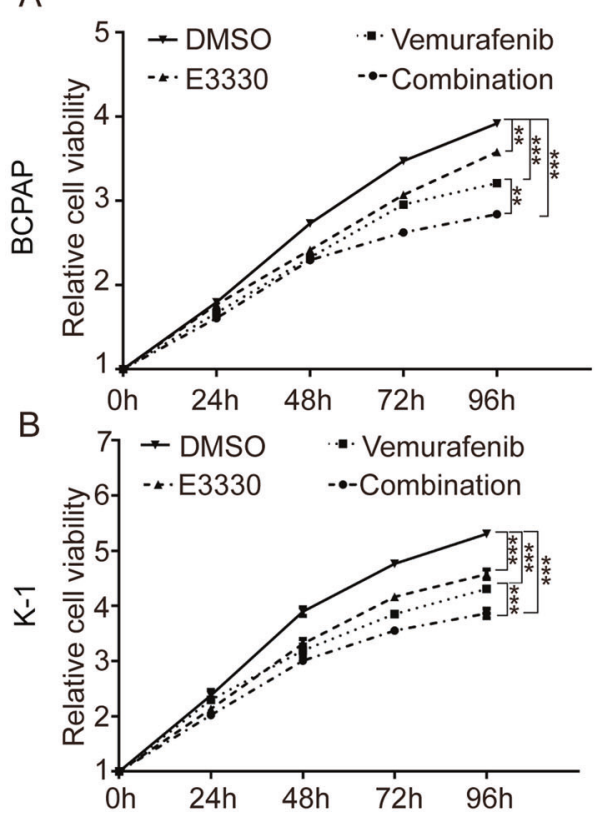

C

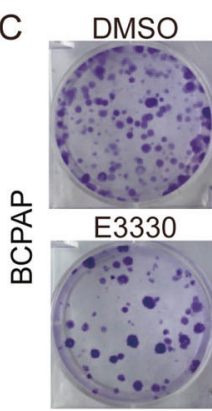

D
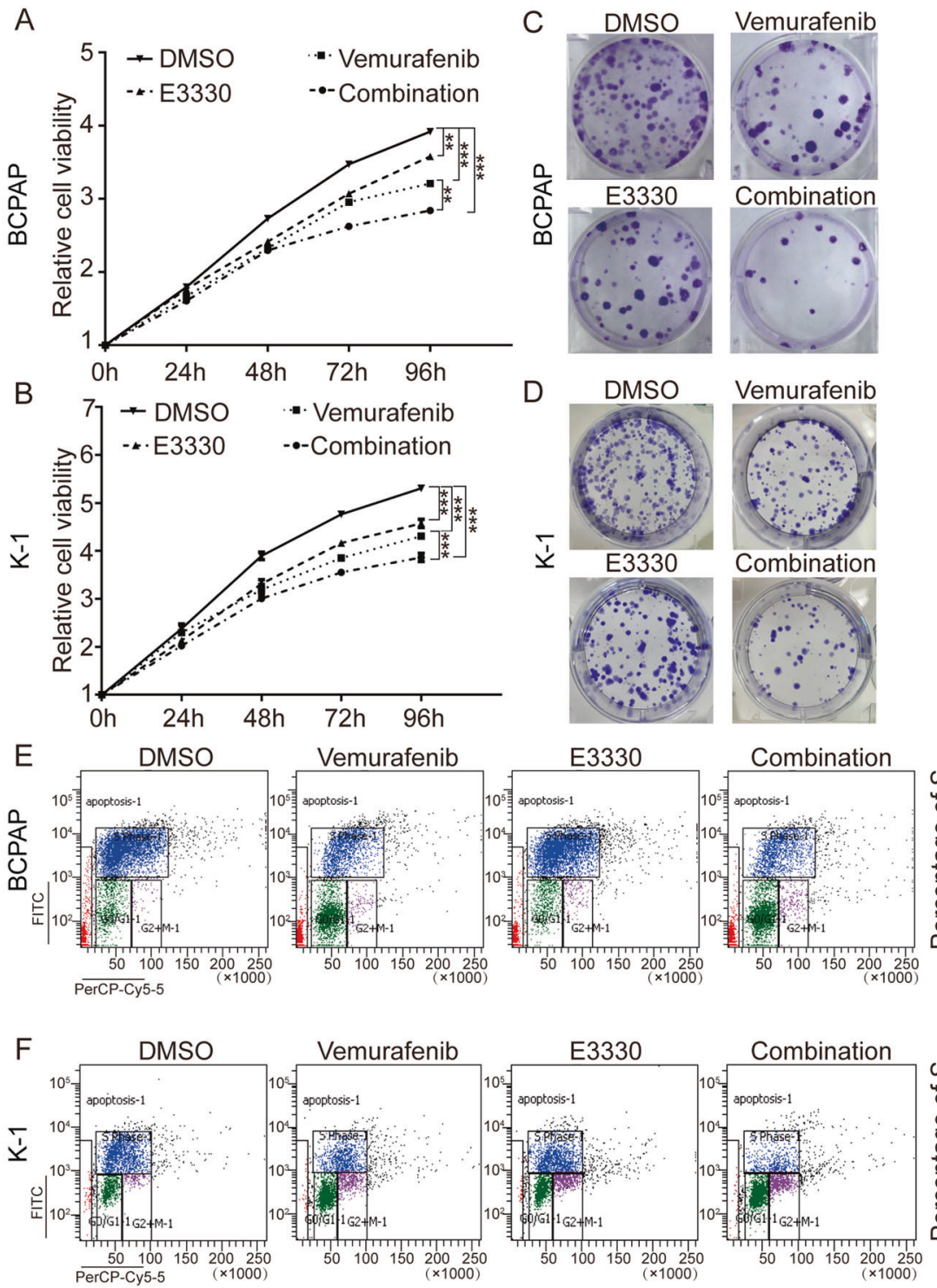

Vemurafenib

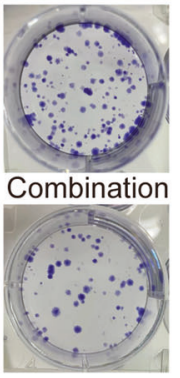

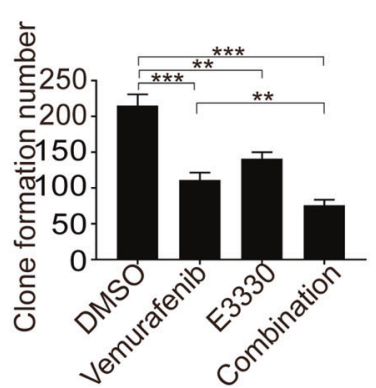

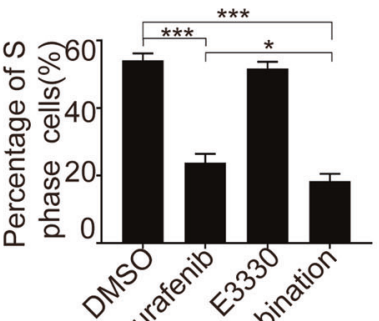

1ents
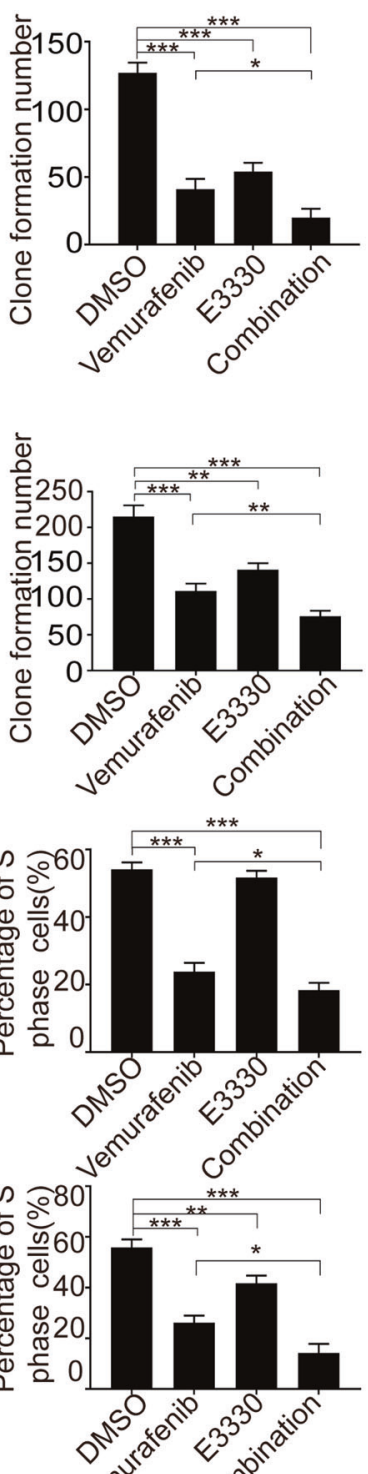

jes $0^{0}$
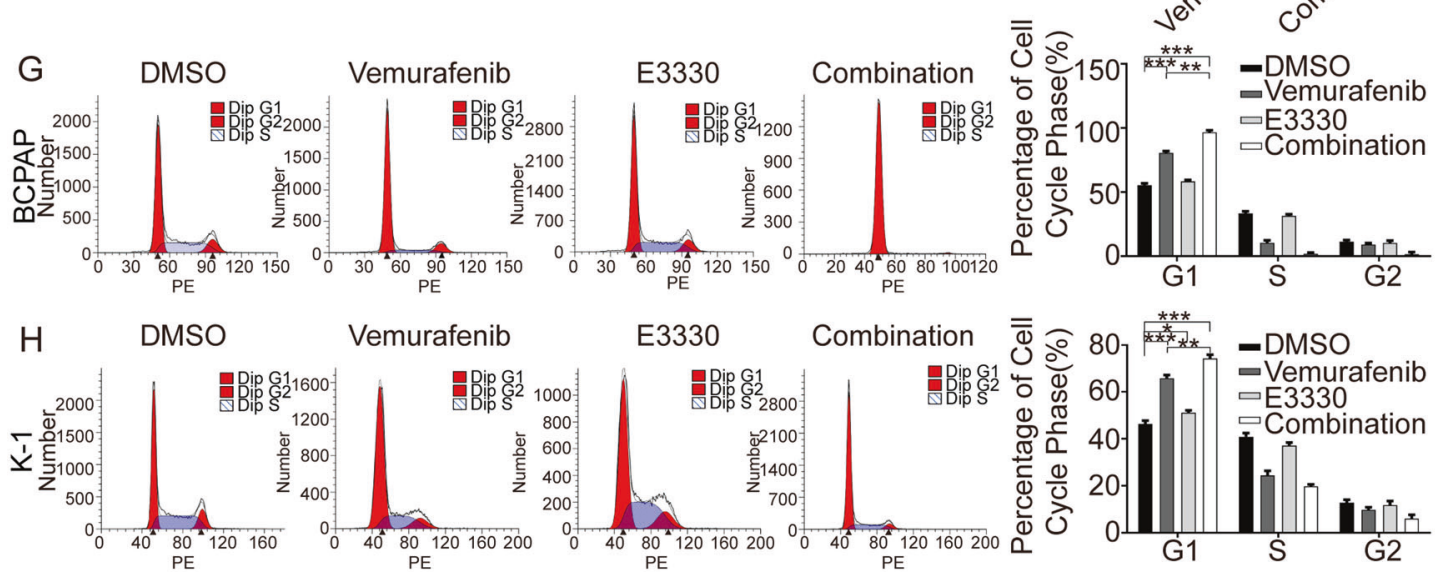

Fig. 3 E3330 enhanced the antiproliferative capacity of vemurafenib in BCPAP and K-1 cells. A, B Cell viability detection by a CCK-8 assay was used to quantify cell proliferation in BCPAP and K-1 cell lines. C, D Cell proliferation detection by colony-formation assay in BCPAP and K-1 cell lines. E, F Cell division detection by BrdU assay in BCPAP and $K-1$ cell lines. $\mathbf{G}, \mathbf{H}$ The cell cycle distribution detection by flow cytometric assay in BCPAP and K-1 cell lines. BCPAP and K-1 cell lines were pretreated with DMSO, vemurafenib $(10 \mu \mathrm{M}), \mathrm{E} 3330(50 \mu \mathrm{M})$, and combination $\left(10 \mu \mathrm{M}\right.$ vemurafenib $+50 \mu \mathrm{M}$ E3330), respectively. ${ }^{*} P<0.05,{ }^{* *} P<0.01,{ }^{* * *} P<0.001$. 

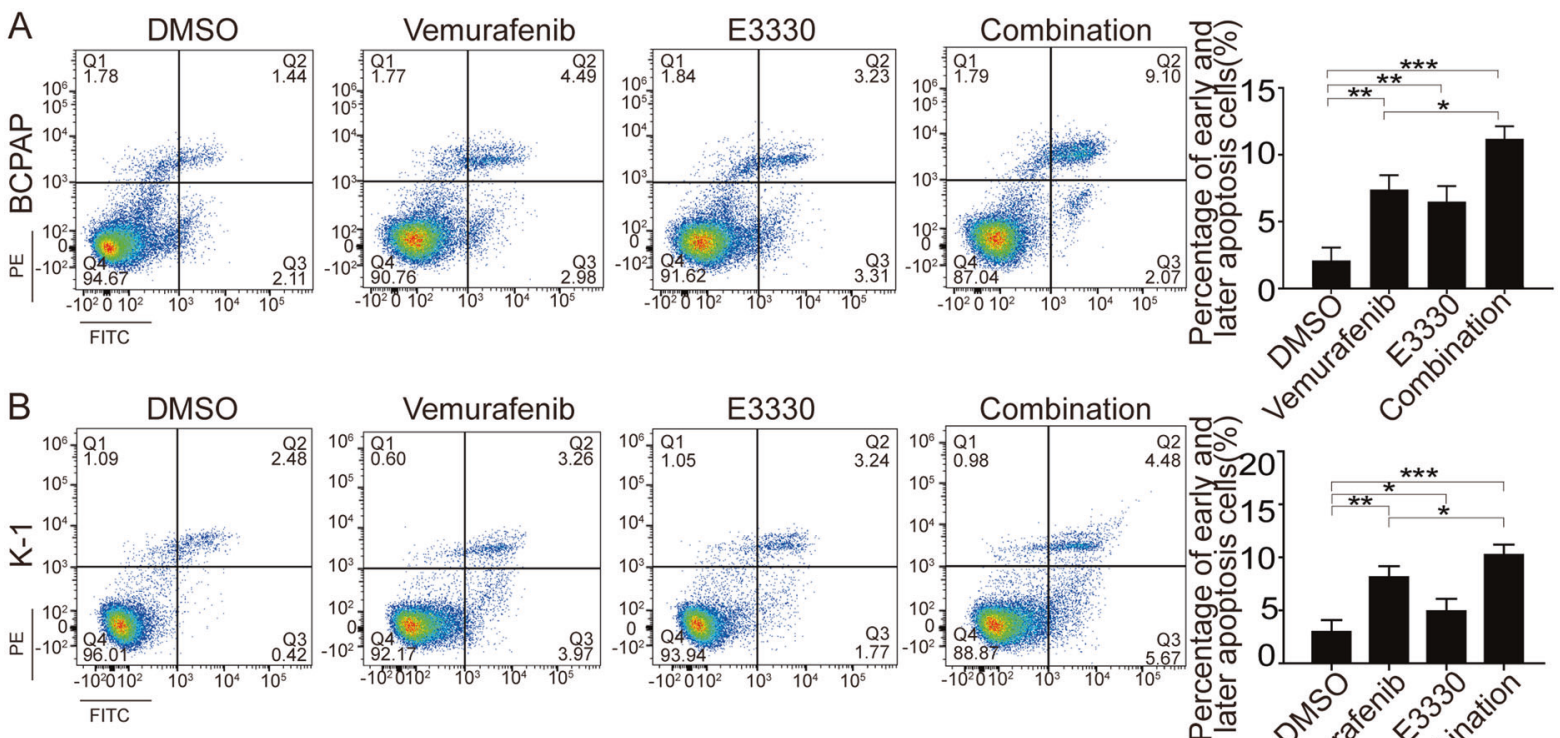
jêt
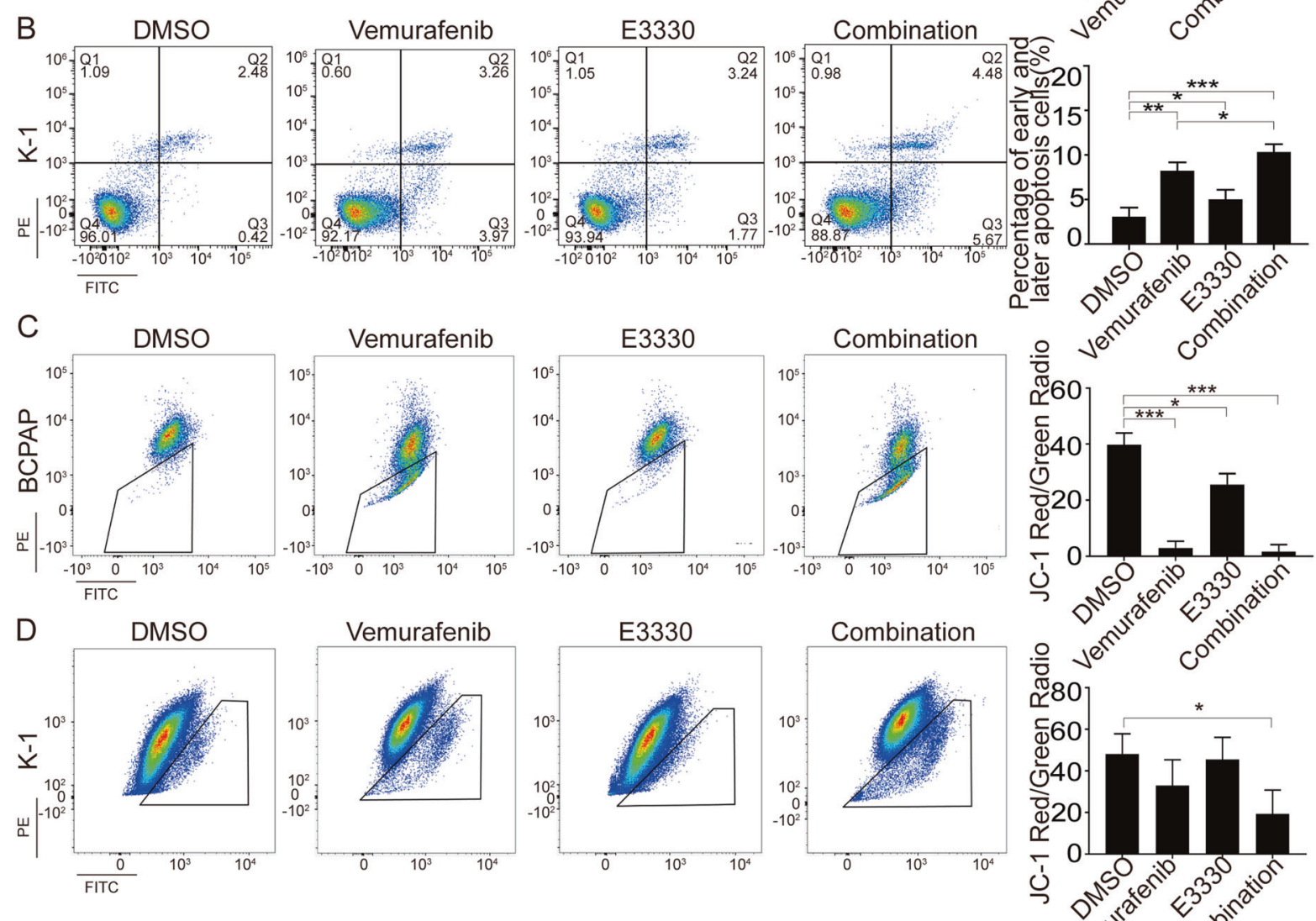

E

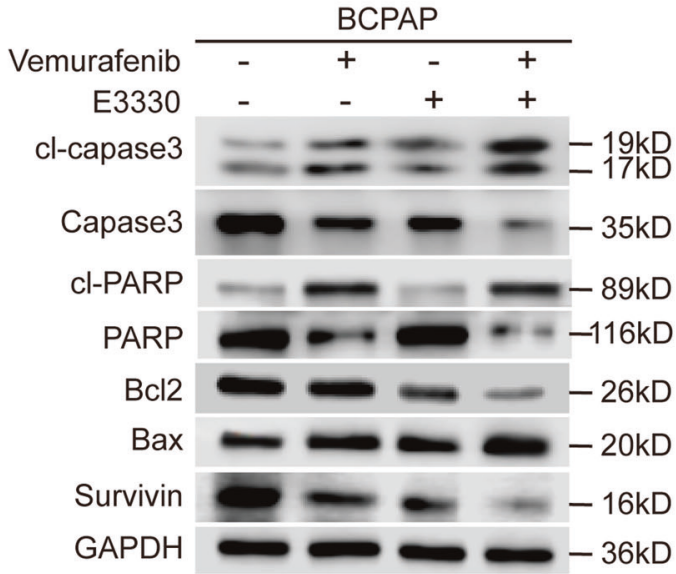

$\mathrm{F}$
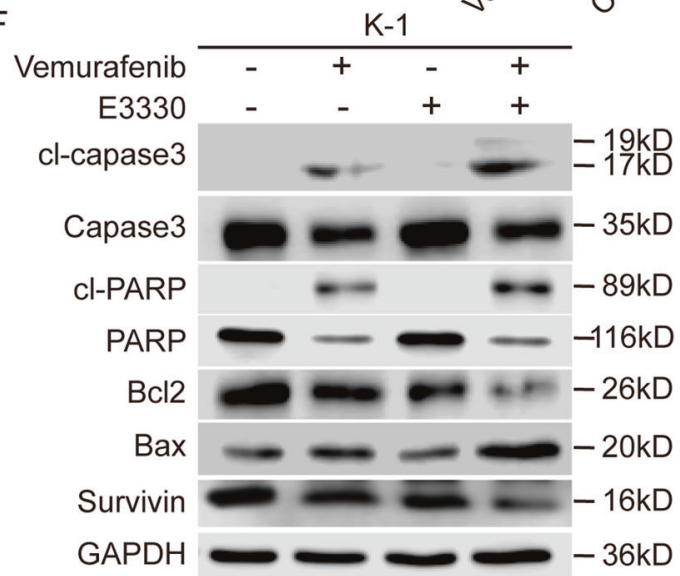

Fig. 4 E3330 enhanced the proapoptotic capacity of vemurafenib in BCPAP and K-1 cells. A, B The cell apoptosis rate measured by flow cytometric analysis in BCPAP and K-1 cell lines. C, D The mitochondrial membrane potential evaluation by detecting JC-1 content in BCPAP and K-1 cell lines. E, F Mitochondrial apoptosis pathway-associated protein expression detection by western blot assay in BCPAP and K-1 cell lines. BCPAP and K-1 cell lines were pretreated with DMSO, vemurafenib $(10 \mu \mathrm{M})$, E3330 $(50 \mu \mathrm{M})$, and combination $(10 \mu \mathrm{M}$ vemurafenib $+50 \mu \mathrm{M}$ E3330), respectively. ${ }^{*} P<0.05,{ }^{* *} P<0.01,{ }^{* * *} P<0.001$. 

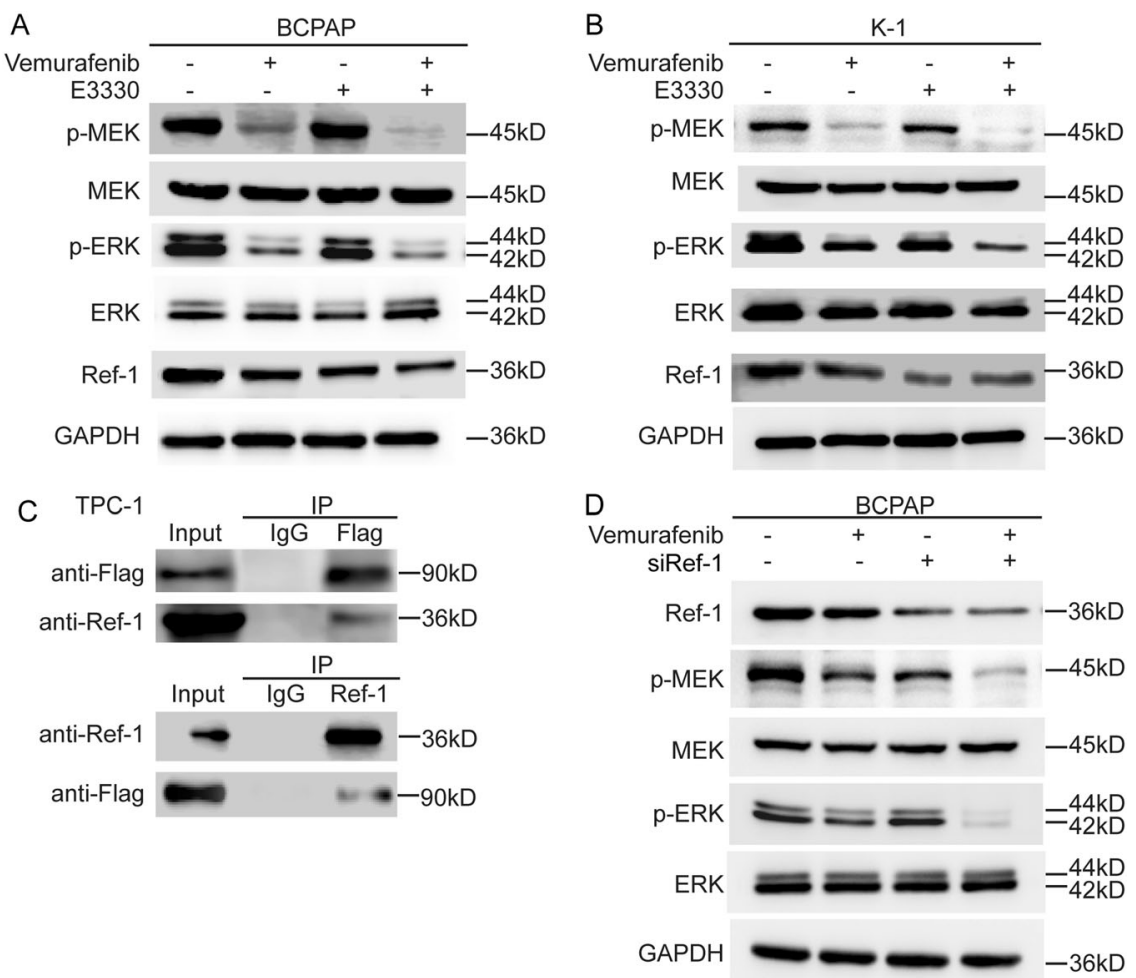

Fig. 5 Ref-1 promoted the maintenance of mutant BRAF function. A, B Western blot detection of MAPK pathway activation in DMSO, vemurafenib $(10 \mu \mathrm{M})$, E3330 $(50 \mu \mathrm{M})$, and combination $(10 \mu \mathrm{M}$ vemurafenib $+50 \mu \mathrm{M}$ E3330) groups. C The mode of action of Ref-1 and BRAF determination by a Co-IP assay using TPC-1 cells with a BRAF ${ }^{\mathrm{V} 600 \mathrm{E}}$ Tet-on system. D MAPK pathway activation detection by western blot assay after small interfering RNA targeting of Ref-1 with vemurafenib treatment.

Senescence was involved in the dual-suppression outcome of Ref-1 and BRAF through an overloaded autophagic flux effect A number of studies [23-26] have shown that vemurafenib can promote the occurrence of protective autophagy while inducing limited apoptosis, which is an important reason for treatment resistance. In fact, the duration and intensity of the autophagosome signal can cause completely different outcomes that determine whether tumors survive or die [27]. To verify the autophagic phenomenon induced by vemurafenib treatment and identify the final trend in autophagy, we observed the formation and fusion of autophagosomes, lysosomes, and the distribution and expression level of the common autophagy marker in the meanwhile. In the vemurafenib group and E3330 group, autophagosomes, lysosomes, and other organelles fused into autophagolysosomes at different stages of formation. And in the combination group, we found that following the increase of phagocytosis and fusion, more and larger vacuoles were formed in the cells, occupying most of the cytoplasmic space, and there were few organelles left in the cells (Fig. 6A). In addition, enhanced autophagy induced by vemurafenib was notably present, and combined treatment with vemurafenib and the Ref-1 inhibitor strikingly induced LC3B, p62 colocalization and accumulation, AMP-activated protein kinase (AMPK) activation, ATG5 expression increasing in the BCPAP and K-1 cell lines, which indicated an incomplete or excessive autophagy process (Fig. 6B, C). Research has reported that basal autophagy is essential to maintain stem cell quiescence by preventing aging, while the accumulation of autophagic substrates can cause cell senescence and death $[28,29]$. Subsequently, we performed senescence staining and $\mathrm{\gamma H} 2 \mathrm{AX}$ marking in BCPAP and $\mathrm{K}-1$ cell liens after single or combined treatment with the two inhibitors. A highly significant senescence phenotype that occurred in the combined treatment strategy was observed (Fig. 6D, E). Then, HCQ (hydroxychloroquine), a lysosomal autophagy inhibitor that can block autophagy substrate conversion, was used to further validate the senescence phenotype caused by the impaired autophagy process. (Fig. 6F, G).

The Ref-1 redox inhibitor enhances the sensitivity of PTC cells to vemurafenib treatment in vivo

Xenogeneic subcutaneous tumor model experiments showed that combined vemurafenib and Ref- 1 inhibitor treatment significantly inhibited the growth and progression of xenogeneic subcutaneous tumors (Fig. 7A). Moreover, the combination group showed the effect of complete reduction for PTC, and the combination strategy is expected to enable complete remission of early-stage tumors (Fig. 7B). Increased rate of body weight and hematoxylin-eosin (H\&E) staining of liver and kidney tissue samples showed a reliable safety coefficient (Fig. 7C, D). IHC staining of tumor tissue samples showed decreased Ki-67 and p-ERK expression and increased cl-caspase 3 and $\mathrm{yH} 2 \mathrm{AX}$ expression (Fig. 7E).

In lung metastasis animal experiment analysis, compared with single-drug treatment, combined vemurafenib and Ref-1 inhibitor treatment significantly inhibited the growth of metastatic tumors and tumor progression (Fig. 7F). Additionally, in addition to lung metastasis, a liver metastasis case occurred in both the control group and the vemurafenib group during drug administration, indicating that E3330 might have a superior inhibitory effect on tumor metastasis (Fig. 7G). IHC staining of lung metastatic tumor tissue sections indicated decreased cell proliferation number as determined by Ki- 67 staining; depressed cell metastasis ability, as determined by Vimentin staining; and overloaded autophagy flux, as determined by LC3B and p62 staining (Fig. 7H). These results were consistent with the results of the in vitro cell experiments, indicating that E3330 could exert sensitization and synergistic effects in combination with vemurafenib on tumor progression in vivo. 

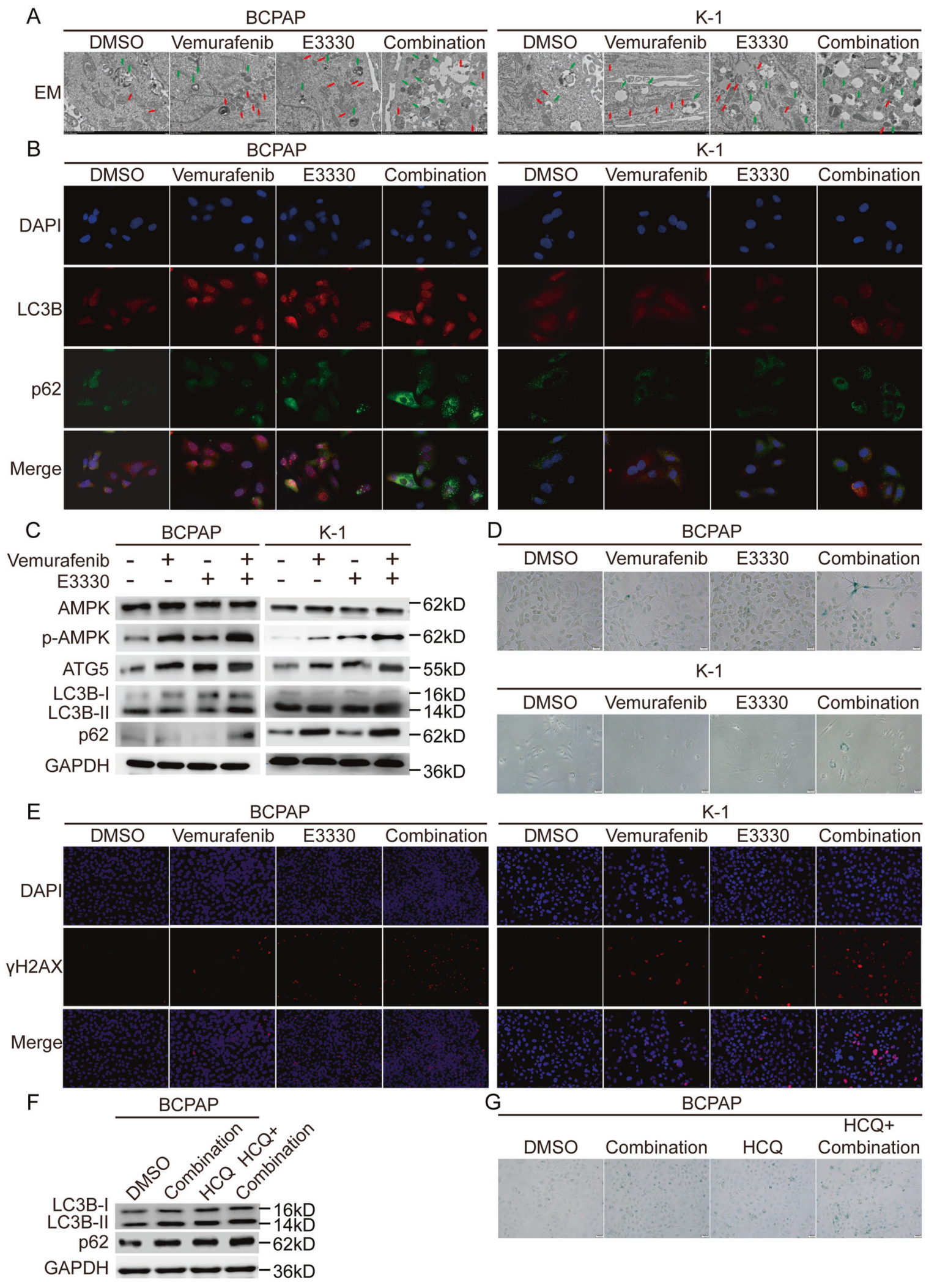

\section{DISCUSSION}

In addition to the high recurrence rate and reduced survival rate, the dedifferentiation of thyroid cells and decline in functional gene expression are also closely related to BRAF mutation [30]. BRAF ${ }^{\mathrm{V} 600 \mathrm{E}}$, which accounts for $90 \%$ of BRAF mutations in human cancers [31] and has high catalytic activity alone, does not require dimerization for function $[32,33]$, which is one of the important reasons for the high degree of malignancy of BRAF-mutant PTC. As a competitive inhibitor, vemurafenib selectively binds to the ATP (adenosine 
Fig. 6 Combined Ref-1 redox inhibitor and vemurafenib treatment induced autophagic flow overload and caused senescence in BCPAP and K-1 cells. A Representative electron micrographs of BCPAP and K-1 cell lines. The green arrows indicate lysosome and the red arrows indicate autophagosome. B Immunofluorescence detection of the autophagic substrates LC3B and p62 in BCPAP and K-1 cell lines. C Western blot detection of the autophagic substrates LC3B and p62 in BCPAP and K-1 cell lines. D Cellular senescence detection by $\beta$-galactosidase staining in BCPAP and K-1 cell lines. E Immunofluorescence detection of $\gamma \mathrm{H} 2 \mathrm{AX}$ in BCPAP and K-1 cell lines. F Western blot detection of the autophagic substrates LC3B and p62 in BCPAP and K-1 cell lines. G Cellular senescence detection by $\beta$-galactosidase staining in BCPAP and K-1 cell lines. BCPAP and K-1 cell lines in A-E were pretreated with DMSO, vemurafenib $(10 \mu \mathrm{M})$, E3330 $(50 \mu \mathrm{M})$, and combination $(10 \mu \mathrm{M}$ vemurafenib $+50 \mu \mathrm{M}$ E3330), respectively. BCPAP and K-1 cell lines in F, G were pretreated with DMSO, combination (10 $\mu \mathrm{M}$ vemurafenib + $50 \mu \mathrm{M}$ E3330), $\mathrm{HCQ}$, and combination $+\mathrm{HCQ}$, respectively.

triphosphate)-binding site of the kinase BRAF, thereby reducing ATP entry to block MAPK pathway activation [31].

In recent years, targeted therapies for PTC have changed focus from inhibiting tumor neovascularization to targeting specific gene mutations. In a preliminary analysis, we observed lower sensitivity to vemurafenib in PTC patients and cell lines than in melanoma patients and a melanoma cell line. At present, according to published reports, there are two main reasons for PTC patients developing resistance to vemurafenib therapy: one is the compensatory or bypass activation of MAPK pathway-related proteins, and the other is the heterogeneity of cells with various genetic backgrounds in the same tumor tissue and different types of tumor cells [34]. The former mechanism is mainly classified as acquired resistance and has been revealed to involve multifarious signaling pathways and occur in various tumors. The latter is generally considered intrinsic resistance, but the mechanism remains unclear.

In fact, the thyroid is an organ that needs to synthesize oxidative thyroid hormones, and this process inevitably generates a large amount of reactive oxygen free radicals. A number of studies have shown that TC tissues have higher levels of oxidants than that of normal tissues $[35,36]$. The production of ROS in TC mainly derives from NADPH oxidase and mitochondria, in which NOX4 is the only NADPH oxidase with constitutive ROS generation activity and directly depends on its gene expression [35, 37]. Considering that Ref- 1 is a classic redox factor in preventing cells from being damaged by high concentrations of reactive oxygen species, we analyzed the correlation between BRAF mutation, APEX1 expression level, and NOX4 expression level in PTC, which represents higher levels of ROS to some extent. The results showed the expression level of NOX4 is positively correlated with the mutation state of BRAF and the expression level of APEX1. We speculated that in BRAF-mutated PTC, Ref- 1 by BRAF mutations accompanied by high levels of ROS may be an important redox balance system caused to activate.

E3330, as a specific inhibitor of Ref- 1 redox function, exerts an important antitumor effect on various tumors [38-40]. In this study, we proved that E3330 positively enhanced the treatment sensitivity and antitumor capacity of vemurafenib in PTC both in vitro and in vivo. We ultimately revealed another conceivable mechanism by which BRAF can maintain its activation status in the context of inhibitor treatment through the binding effect of the Ref- 1 domain and the conformational change in Ref- 1 caused by E3330 dissociating Ref-1 from the BRAF protein, which provides an opportunity for vemurafenib to enter the active pocket, thereby enhancing the inhibitory effect of vemurafenib; however, unfortunately, the definite mechanism is still not clear. Understanding the binding site and mode of action between Ref- 1 and BRAF may provide a more useful therapeutic schedule. In addition, as we suppressed the expression of endogenous full-length Ref-1, we cannot exclude the possibility that the DNA repair activity of Ref-1 may also contribute to its sensitivity-promoting effect supporting vemurafenib treatment efficacy, and redox-targeting studies need to be performed in the future.

Currently, many mechanisms of vemurafenib resistance have been reported, among which the strong and acknowledged autophagy reaction caused by vemurafenib treatment clearly reduces the antitumor effect of this drug [23, 24, 41]. Autophagy can protect cancer cells by eliminating damaged organelles and recovering degradation products in normal cells, which may be the cause of resistance mediated via autophagy [42]. Numerous signaling pathways take part in the regulation of the autophagy process, such as the PI3K/AKT/mTOR pathway [43, 44], LKB1 (liver kinase B1)/AMPK/mTOR pathway [45-47], MAPK pathway [48, 49], p53 pathways (genotoxic stress) [50, 51], and so on. A close connection between vemurafenib and autophagy activated by the AMPK pathway has been reported many times. Sueda et al. [45] found that $\mathrm{BRAF}^{\mathrm{V} 600 \mathrm{E}}$ inhibitor treatment induced protective autophagy through AMPK activation, resulting in an attenuated drug effect on colorectal cancer cells. Niehr et al. [52] demonstrated that phosphorylated and activated AMPK after vemurafenib treatment could induce protective autophagy as our results showed.

Initially, in yeast, cells only used the autophagy program to fulfill autologous metabolic needs or renew certain organelles, thereby maintaining the vitality of cells suffering from nutritional deterioration [53]. However, in higher eukaryotes, autophagy plays much more complicated and multifunctional roles in regulating cell survival and death, especially in cancer cells $[27,29,54,55]$. The commonly studied proteins closely related to the autophagy program mainly include LC3B and p62 [55-57], and their expression represents the differential autophagy status and output. The process of autophagosome fusion with acidic endosomes/lysosomes to form autolysates is defined as "autophagic flux" [54], which is one particular vital part of the process in the regulation of cell life. If contents are not cleared, scilicet eliminated or processed through autophagic flux, they accumulate and damage the cell, resulting in cellular senescence [29]. We observed in this study that combined inhibition with the two drugs studied appeared to be the phenomenon that enhanced AMPK activation, increased autophagy, which eventually led to the accumulation of cellular metabolic substrates or excessive consumption leading to senescence. Although senescence is often considered to be a temporary quiescent state to escape the immune system killing or to produce carcinogenic effects on surrounding cells through senescence-associated secretory phenotype (SASP), senescence induced by excessive autophagy and subsequent imbalance between energy consumption and supply caused by the autophagic turnover disorder of cellular constituents may lead to irreversible death of cells. But so far, the causal relationship between autophagy and senescence is still inconclusive. And in the combination group, we found that following the increase of phagocytosis and fusion, more and larger vacuoles were formed in the cells, occupying most of the cytoplasmic space, and there were few organelles left in the cells. These results indicate that autophagy and senescence are related events. The induction of senescence partly depends on the activation of autophagy, and excessive autophagy can lead to irreversible senescence events.

Several studies $[25,41,44]$ have reported that dual inhibition of MAPK and autophagy caused by a MAPKi can specifically improve clinical efficacy in BRAF ${ }^{\mathrm{V} 600 \mathrm{E}}$ tumors; however, excessive 


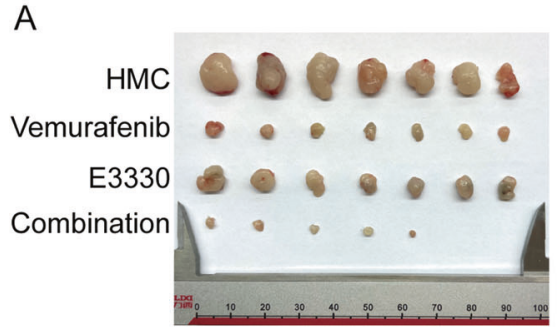

C

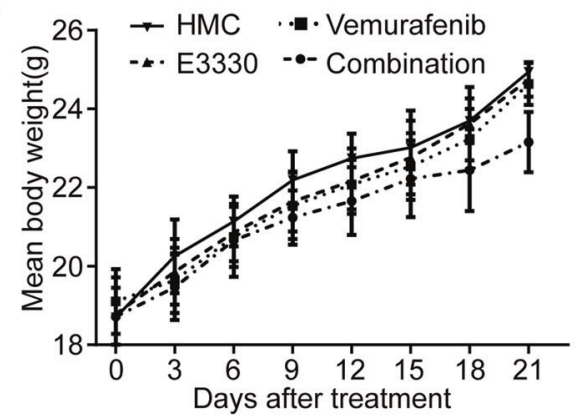

$E$

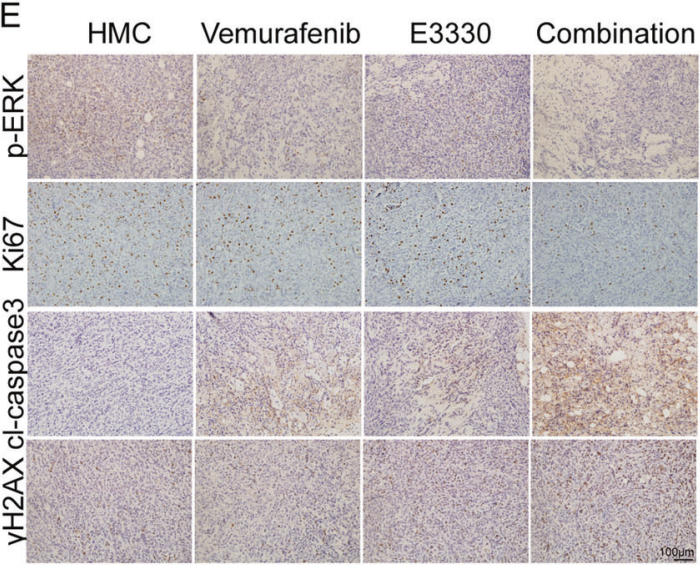

G
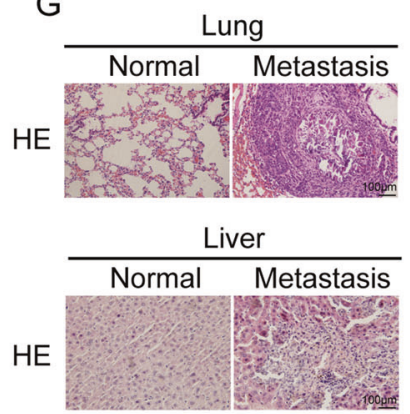

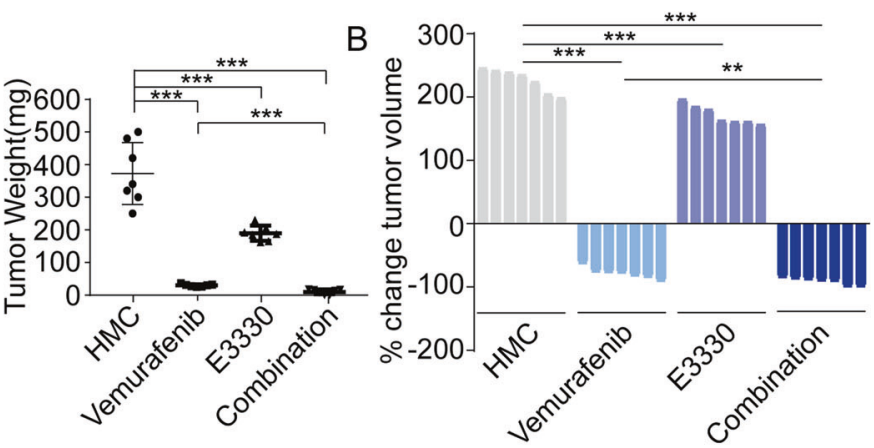

D HMC Vemurafenib E3330 Combination
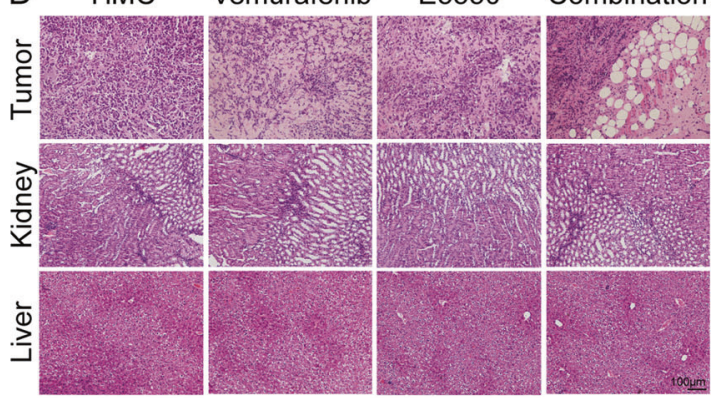

HE $(200 \times)$

$\mathrm{F}$

0 days
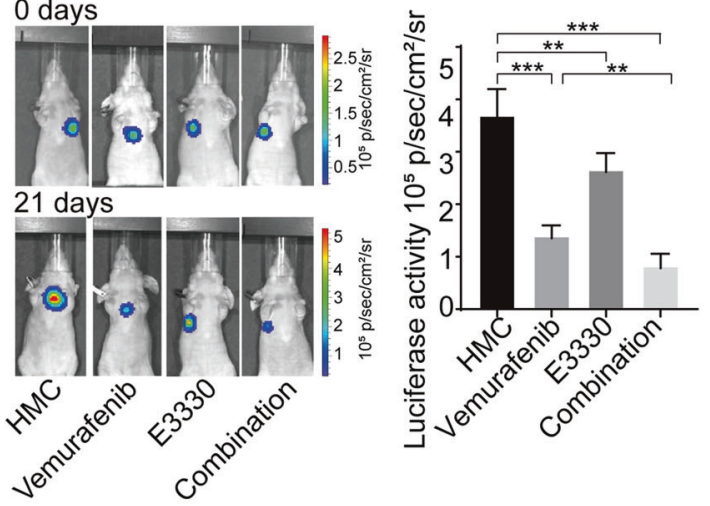

$\mathrm{H}$

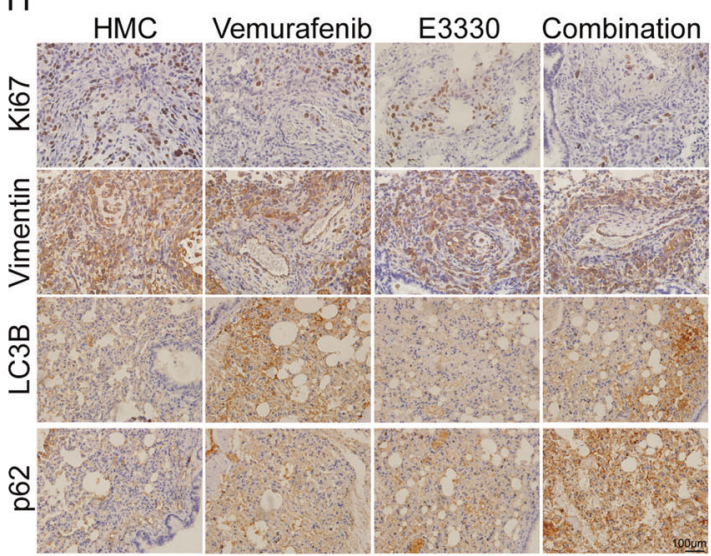

inhibiting Ref- 1 could further enhance the competitive blockade effect of vemurafenib on the constitutively active BRAF binding site, it is conceivable that the AMPK/mTOR pathway was further activated, thereby enhancing autophagy activation. Yuan et al. [58] combined AMPK activators and a BRAFi to perform 
Fig. 7 Vemurafenib combined with E3330 treatment offered a relatively promising therapeutic strategy in vivo. A Representative images of dissected mouse subcutaneous tumors after HMC, vemurafenib, E3330, or combination (vemurafenib + E3330) treatment for 21 days. B The weights of mouse subcutaneous tumors after different treatments for 21 days. C Change in tumor volume after HMC, vemurafenib, E3330, or combination (vemurafenib + E3330) treatment for 21 days. D The body weight changes of mice measured every 3 days after different treatments. E Representative H\&E staining of tumors, livers, and kidneys after different treatments. F Representative immunohistochemical staining for p-ERK, Ki-67, cl-caspase3, and $\gamma \mathrm{H} 2 \mathrm{AX}$ after different treatments. G Representative images of mouse metastatic tumors after HMC, vemurafenib, E3330, or combination (vemurafenib + E3330) treatment for 21 days. $\mathbf{H}$ Representative H\&E staining of metastatic tumors in the lungs and livers. I Representative immunohistochemical staining for Ki-67, Vimentin, LC3B, and p62 after different treatments. ${ }^{* * P}<0.01,{ }^{* * *} P$ $<0.001$.

experiments in melanoma, and they uncovered overlapping AMPK activation with superior antitumor effects for vemurafenib, which supports our conclusion to some extent.

\section{CONCLUSION}

In this study, we found that the $\mathrm{BRAF}^{\mathrm{V} 600 \mathrm{E}}$ mutation upregulated the expression of Ref- 1 in PTC patients, which might be one important reason for intrinsic resistance to BRAFi treatment. Combined treatment with vemurafenib and a Ref- 1 redox inhibitor could enhance the antitumor capacity of vemurafenib by abolishing the maintenance effect on the BRAF protein and overloading autophagic flux both in vitro and in vivo; this approach is a potential strategy for BRAF-mutant PTC treatment.

\section{MATERIALS AND METHODS}

\section{Clinical data and tissue samples}

Formalin-fixed tissue samples from 178 PTC patients with complete clinicopathological information embedded in paraffin were made into 4 tissue microarrays, and $5-\mu \mathrm{m}$ sections were cut. And 16 pairs of matched fresh tissues of PTC carcinoma and adjacent normal thyroid follicular tissue were collected in 2015 for total RNA and protein extraction. Informed consent was obtained from each patient to allow their information to be used. The research was performed with the approval of the Ethics Committee of Tianjin Medical University Cancer Institute and Hospital.

\section{Cell culture}

The human TC cell lines K-1, BCPAP, and TPC-1 involved in this study all have STR identification certificates and are tested for mycoplasma contamination. BCPAP (BRAF ${ }^{\mathrm{V} 600 \mathrm{E}}$ mutation), $\mathrm{K}-1$ (BRAF ${ }^{\mathrm{V} 600 \mathrm{E}}$ mutation), and TPC-1 $\left(\mathrm{BRAF}^{\mathrm{wt}}\right)$ human papillary thyroid carcinoma cells were purchased from Bestbay. BCPAP and TPC-1 cells were cultured in RPMI 1640 Medium (Gibco, c11875500bt) supplemented with $10 \%$ fetal bovine serum (Eallbio, u16001dc), 1\% L-glutamine (Gibco, 25030081), 1\% nonessential amino acid solution (Gibco, 11140050) and $1 \%$ penicillinstreptomycin (Gibco, 10378016, $100 \mathrm{U} / \mathrm{mL}$ penicillin, $100 \mu \mathrm{g} / \mathrm{mL}$ streptomycin). K-1 cells were cultured in Dulbecco's Modified Eagle Medium (Gibco, c11995500bt) with the same supplementation used for BCPAP cells. The cells were maintained in an incubator set to $37^{\circ} \mathrm{C}$ with $5 \% \mathrm{CO}_{2}$.

\section{Drugs, reagents, and antibodies}

Vemurafenib (a novel small molecular inhibitor against V600E, V600D, and V600R mutant cell lines, competing with ATP for the binding domain of BRAFV600E-mutated monomer) and E3330 (a quinone derivative, binding to a partially unfolded state of Ref- 1 and increasing the formation of disulfide bonds between cysteine residues to convert Ref- 1 into a folded state for inhibition of its redox function) were purchased from Selleck Chemical (Shanghai, China) and diluted in dimethyl sulfoxide (DMSO). An anti-GAPDH antibody was purchased from Genetex (GTX100118). Anti-Ref1 (ab202894) and anti-Actin (ab8226) antibodies were purchased from Abcam. Anti-Caspase3 (\#9664), anti-cleaved-Caspase3 (\#9602), anti-PARP (\#9532), anti-cleaved-PARP (\#5625), anti-p62 (\#23214), anti-LC3B (\#3868), anti-E-cadherin (\#14472), anti-Vimentin (\#5741), anti-Bcl2 (\#15071), antiBax (\#5023), anti-Ki67 (\#9449), anti-MEK (\#4694), anti-p-MEK (\#9127), antiERK (\#4695), and anti-p-ERK (\#4370) antibodies were purchased from Cell Signaling Technology. Anti-Survivin (AB3610) and anti-Flag (F1804) antibodies were purchased from Sigma.

\section{Immunohistochemistry}

The expression of Ref-1 in papillary thyroid carcinoma was detected using conventional IHC. Lung metastatic tumor and liver sections from animals were stained with H\&E for routine histological examination and morphometric analysis or IHC stained.

For H\&E staining, briefly, tissue sections on coated slides were dewaxed with xylene and gradient alcohol after being incubated in an oven at $70^{\circ} \mathrm{C}$ for $1 \mathrm{~h}$, counterstained with H\&E, dehydrated, and covered.

For IHC staining, briefly, tissue sections were dewaxed as indicated in the $\mathrm{H} \& \mathrm{E}$ staining procedure and then subjected to antigen retrieval by boiling in $10 \mathrm{mM}$ sodium citrate $(\mathrm{pH} 6.0)$ at $130^{\circ} \mathrm{C}$ for $3 \mathrm{~min}$. The slides were then pretreated with a $3 \%$ solution of hydrogen peroxide for $30 \mathrm{~min}$, rinsed, and incubated with $5 \%$ normal goat serum for $20 \mathrm{~min}$ as a blocking agent. The sections were incubated with a mouse anti-Ref- 1 antibody (1:800; Abcam) at $4{ }^{\circ} \mathrm{C}$ overnight. On the next day, the slides were washed in PBS and incubated with a secondary antibody for $30 \mathrm{~min}$ at room temperature. All steps were preceded by rinsing the sections with PBS ( $\mathrm{pH}$ 7.6). The chromogen was 3,3-diaminobenzidine (DAB). Hematoxylin was used for counterstaining, and dehydrated gum was used as the sealant; the slides were then observed, scored, and imaged. Staining intensity was scored by three professional pathologists in a double-blind way and criteria as follows: $0(-), 1(+), 2(++)$, and $3(+++)$. The degree of staining was categorized as 0 ( $0 \%$ staining), 1 (1-30\% staining), 2 (31-60\% staining), 3 $(61-80 \%$ staining), and $4(81-100 \%$ staining). The final score was determined by multiplying the staining intensity score and the staining degree score, ranging from 0 to 12 . Samples with a final score of less than 3 were considered to have low expression, while those with a score of 4-12 were considered to have high expression.

\section{Quantitative real-time PCR}

Total RNA was extracted from PTC cells and fresh tissue using TRIzol reagent (Life Technologies), and $2 \mu \mathrm{g}$ of RNA was reverse transcribed into cDNA by using the High-Capacity CDNA Reverse Transcription Kit (Applied Biosystems). The CDNA was then used as a template for exponential amplification using SYBR Green/ROX qPCR Master Mix (Thermo Scientific). $A C T B$ was used as an internal reference. The primers sequences of APEX 1 and ACTB were as follows respectively: 5'-CAATACTGGTCAGCTCCTTCG-3' and 5'-TGCCGTAAGAAACTTTGAGTGG-3'; 5'-GGAGAGATTGGCTTTCCTGGAC$3^{\prime}$ and $5^{\prime}$-CCTCATGCCAAATCCAAGGCTG-3'.

\section{Western blot analysis}

Cells were cultured in 6-well plates at a density of $5 \times 10^{5} / \mathrm{mL}$ per well and then treated with vemurafenib $(10 \mu \mathrm{M})$ or/and E3330 $(50 \mu \mathrm{M})$ for $24 \mathrm{~h}$. The cells were washed with cold PBS three times and lysed in a RIPA solution (Solarbio LIFE SCIENCES) with the protease inhibitor phenylmethylsulfonyl fluoride (PMSF, Solarbio LIFE SCIENCES) $(1 \mathrm{mM})$ for $30 \mathrm{~min}$ on ice. The supernatants were collected after cell lysates were centrifuged at $12,000 \mathrm{rad}$ for $15 \mathrm{~min}$ at $4^{\circ} \mathrm{C}$. The protein concentrations were quantified using the BCA Protein Assay (Solarbio LIFE SCIENCES) according to the manufacturer's instructions. Equal amounts $(30 \mu \mathrm{g})$ of total protein were separated by sodium dodecyl sulfate-polyacrylamide gel electrophoresis $(10-12 \%)$ and transferred to a $0.45-\mu m$ PVDF membrane. After blocking with $5 \%$ bovine serum albumin (BSA) in TBST buffer for $2 \mathrm{~h}$ at room temperature, the membranes were incubated with a primary antibody at $4^{\circ} \mathrm{C}$ overnight. The membranes were washed three times with TBST buffer and then incubated with peroxidase (HRP)-conjugated secondary antibody for $1 \mathrm{~h}$ at room temperature. Specific antibody binding was detected with the Chemiluminescence Kit (Millipore, Plano, TX, USA). Fluorescent signals were detected by a luminescent image analyzer (C-Digit, Gene Company Limited, China). 


\section{Cell viability and colony formation assays}

A Cell Counting Kit-8 (CCK-8) assay (Dojindo, Kumamoto, Japan) was used according to the manufacturer's instructions to measure the effects of drugs on the proliferation of papillary thyroid carcinoma cells. Briefly, BCPAP cells were seeded in 96-well cell plates (Corning Inc., Corning, USA) at a density of 1000 cells/well in $200 \mu \mathrm{L}$ of culture medium and grown overnight. The next day, vemurafenib or/and E3330 were added to each well at an appropriate concentration, and cells were incubated for $0,24,48$, 72 , and $96 \mathrm{~h}$. Then, $20 \mu \mathrm{L}$ of CCK-8 reagent was added to each well and incubated for $3 \mathrm{~h}$ at $37^{\circ} \mathrm{C}$. Finally, the absorbance was measured with an enzyme-labeled instrument (Thermo) at $450 / 650 \mathrm{~nm}$. The experiments were repeated at least three times.

For colony formation analysis, BCPAP and $\mathrm{K}-1$ cells were seeded in 6-well cell plates (Corning Inc., Corning, USA) at a density of 500 and 1000 cells/ well in $2 \mathrm{~mL}$ of culture medium, respectively, and then treated with vemurafenib or/and E3330 at an appropriate concentration for $24 \mathrm{~h}$. In the drug treatment groups, the medium was replaced with a fresh medium after 14 days. Colonies were washed with phosphate-buffered saline (PBS) 3 times, fixed with $4 \%$ paraformaldehyde for $20 \mathrm{~min}$, stained with $0.5 \%$ crystal violet for $15 \mathrm{~min}$ at room temperature, and then counted.

\section{Flow cytometric analysis}

For analysis of apoptosis, BCPAP and K-1 cells were treated with $10 \mu \mathrm{M}$ vemurafenib, $50 \mu \mathrm{M}$ E3330, or their combination in 6-well plates for $24 \mathrm{~h}$. Apoptosis rates were assessed by flow cytometry after staining with Annexin V/propidium iodide (BD Pharmingen, Franklin Lakes, USA). In brief, cells were harvested, washed three times with cold PBS, and resuspended in $100 \mu \mathrm{L}$ of binding buffer at a density of $1 \times 10^{5}$ cells $/ \mathrm{mL}$. Then, $5 \mu \mathrm{L}$ of Annexin $V$ and $5 \mu \mathrm{L}$ of propidium iodide were added to the cells and incubated for $15 \mathrm{~min}$ in the dark at room temperature. Then, flow cytometry was performed, and the results were reported for three independent experiments. After administration of the above-indicated treatments, the cell cycle was also evaluated. In brief, cells were harvested, washed three times in cold PBS, fixed with $70 \%$ ethanol in PBS at $4{ }^{\circ} \mathrm{C}$ overnight, and then incubated with $100 \mu \mathrm{g} / \mathrm{mL}$ RNase A, $0.2 \%$ Triton X-100 and $50 \mu \mathrm{g} / \mathrm{mL}$ propidium iodide for $30 \mathrm{~min}$ at $4{ }^{\circ} \mathrm{C}$ in PBS. The cells were analyzed by flow cytometry (BD Biosciences FACSCanto II, USA). The results were reported for three independent experiments. For BrdU analysis, the FITC BrdU Flow Kit was used (BD Pharmingen, Franklin Lakes, USA). In brief, BCPAP and $\mathrm{K}-1$ cells were treated as described above for $12 \mathrm{~h}$, followed by careful addition of $10 \mu \mathrm{L}$ of BrdU solution ( $1 \mathrm{mM} \mathrm{BrdU}$ in $1 \times \mathrm{PBS}$ ) directly to each $\mathrm{mL}$ of tissue culture medium and incubation of the treated cells for $12 \mathrm{~h}$. The BrdU-pulsed cells were selected, and antibodies to specific cell surface markers were added in $50 \mu \mathrm{L}$ of staining buffer. Then, the cells were washed and fixed before treatment with DNase to expose incorporated BrdU. Finally, BrdU and total DNA were stained with fluorescent antibodies and a 7-AAD solution for cell cycle analysis.

\section{Mitochondrial membrane potential assay}

The JC-1 Assay Kit (Beyotime, Beijing, China) was used to measure alterations in the mitochondrial membrane potential according to the manufacturer's instructions. Cells were seeded in six-well plates at a density of $5 \times 10^{5} / \mathrm{mL}$ and then treated with vemurafenib or/and E3330 at concentrations of $10 \mu \mathrm{M}$ or/and $50 \mu \mathrm{M}$ for $24 \mathrm{~h}$. Then, $100 \mu \mathrm{L}$ of JC-1 staining solution was added to $1 \mathrm{~mL}$ of culture medium and incubated for $20 \mathrm{~min}$ at $37^{\circ} \mathrm{C}$ in a $\mathrm{CO}_{2}$ incubator. The samples were analyzed by flow cytometry, with JC-1 aggregates measured in the FL-2 channel and green fluorescence (JC-1 monomers) measured in the FL-1 channel (BD Biosciences).

\section{Electron microscopy (EM)}

EM was carried out as previously described ${ }^{72}$. Briefly, DMSO- and drugtreated cells were fixed overnight at $4{ }^{\circ} \mathrm{C}$ in $2.5 \%$ glutaraldehyde, before being post-fixed with $1 \% \mathrm{OsO}_{4}$ for $1 \mathrm{~h}$. Cells were then dehydrated in a graded ethanol series and embedded in Agar 100 epoxy resin. After finished slicing, stained first with uranyl acetate followed by lead citrate. Sections were observed and photographed under a Philips CM10 Transmission Electron Microscope.

\section{Immunofluorescence}

Cell slides were prepared before the experiment. After $8 \mathrm{~h}$, the slides containing cells that had migrated were immersed in PBS three times for $3 \mathrm{~min}$ each time. The scaffolds were fixed with $4 \%$ paraformaldehyde for
$20 \mathrm{~min}$, permeabilized with $0.5 \%$ Triton $\mathrm{X}-100$ at room temperature for $15 \mathrm{~min}$, and sealed with $5 \% \mathrm{BSA}$ for $1 \mathrm{~h}$ at room temperature. The blocking solution was absorbed by the absorbent paper, and a sufficient amount of diluted primary antibody was added to each slide and incubated in a humidified box overnight at $4{ }^{\circ} \mathrm{C}$. The next day, the slides were washed 3 times with TBST, and a diluted fluorescent secondary antibody was added, followed by incubation at room temperature for $1 \mathrm{~h}$ in the dark. The slides were washed 3 times using TBST and sealed using $5 \mu \mathrm{L}$ of antifade mounting medium with DAPI (Invitrogen). Images were acquired under a fluorescence microscope.

\section{Cell senescence staining experiment}

A cellular senescence $\beta$-galactosidase staining kit (Beyotime, Beijing, China) was used to measure cellular aging according to the manufacturer's instructions. Cells were plated in 6-well plates and treated with vemurafenib or/and E3330 or/and $\mathrm{HCQ}$ at an appropriate concentration for $24 \mathrm{~h}$. Then, $1 \mathrm{~mL}$ of $\beta$-galactosidase staining fixative was added, and the mixture was fixed at room temperature for $15 \mathrm{~min}$. The cells were washed with PBS, and $1 \mathrm{~mL}$ of staining solution was added per well. The 6 -well plate was sealed with plastic wrap to prevent evaporation and then incubated overnight at $37^{\circ} \mathrm{C}$. The plate was observed under an ordinary light microscope, and images were acquired.

\section{Studies in vivo}

The experimental protocol on nude mice was approved by the Ethics Committee of Tianjin Medical University Cancer Institute and Hospital. All procedures involving animals and their care were conducted in conformity with institutional guidelines in compliance with national and international laws and policies. Female BALB/c nude mice ( 5 weeks old) were purchased from the Tianjin Institute of Health and Environmental Medicine. Mice were randomly divided into control groups and different experimental groups by the random number method. The BCPAP cell line was suspended in PBS and injected into mice (seven mice per group) subcutaneously at a concentration of $1 \times 10^{6}$ cells/mouse to establish a xenograft model and the BCPAP cell line subjected to luciferase labeling were suspended in PBS and injected into mice (five mice per group) via the tail vein at a concentration of $1 \times 10^{6} \mathrm{cells} / \mathrm{mouse}$ to establish lung metastasis animal model. After 2 weeks, the mice were fed $2 \%$ hydroxymethyl cellulose (HMC), $20 \mathrm{mg} / \mathrm{kg}$ vemurafenib in $1 \% \mathrm{HMC}$ or/and $50 \mathrm{mg} / \mathrm{kg}$ E3330 in 1\% HMC once a day for 3 weeks. Subcutaneous tumor and metastatic tumor size were respectively measured every 3 days using vernier caliper and live animal imaging system, and body weight was measured every 3 days. At the final time point ( 3 weeks), the mice were sacrificed, and the tumors, lungs, livers, and kidneys were removed and fixed with $4 \%$ paraformaldehyde.

\section{Statistical analysis}

Statistical analysis was performed using the SPSS version 17.0 software and GraphPad Prism 7.0 software. For measurement data, the values are expressed as the mean \pm SEM of at least three separate experiments. Categorical count data are shown as the number of cases. Student's $t$ test (two-sided) and $x^{2}$ tests were used to calculate the significance of differences between groups. Kaplan-Meier survival analysis was performed for the Ref-1 high- and low-risk groups to evaluate the recurrence of patients. Statistical significance is indicated by $P<0.05$.

\section{DATA AVAILABILITY}

The data sets used and/or analyzed during the current study are available from the corresponding author on reasonable request.

\section{REFERENCES}

1. Proietti I, Skroza N, Michelini S, Mambrin A, Balduzzi V, Bernardini N, et al. BRAF inhibitors: molecular targeting and immunomodulatory actions. Cancers. 2020;12:1823.

2. Patel $H$, Yacoub N, Mishra R, White A, Long $Y$, Alanazi S, et al. Current advances in the treatment of BRAF-mutant melanoma. Cancers. 2020;12:482.

3. Fujimura T, Fujisawa Y, Kambayashi Y, Aiba S. Significance of BRAF kinase inhibitors for melanoma treatment: from bench to bedside. Cancers. 2019;11:1342.

4. Dadu R, Shah K, Busaidy NL, Waguespack SG, Habra MA, Ying AK, et al. Efficacy and tolerability of vemurafenib in patients with BRAF(V600E)-positive papillary thyroid cancer: MD Anderson Cancer Center off label experience. J Clin Endocrinol Metab. 2015;100:E77-81. 
5. Kim KB, Cabanillas ME, Lazar AJ, Williams MD, Sanders DL, llagan JL, et al. Clinical responses to vemurafenib in patients with metastatic papillary thyroid cancer harboring BRAF(V600E) mutation. Thyroid. 2013;23:1277-83.

6. Iravani A, Solomon B, Pattison DA, Jackson P, Kumar AR, Kong G, et al. Mitogenactivated protein kinase pathway inhibition for redifferentiation of radioiodine refractory differentiated thyroid cancer: an evolving protocol. Thyroid. 2019;29:1634-45.

7. Jeong JH, Oh JM, Jeong SY, Lee SW, Lee J, Ahn BC. Combination treatment with the BRAF(V600E) inhibitor vemurafenib and the $\mathrm{BH} 3$ mimetic navitoclax for BRAFmutant thyroid carcinoma. Thyroid. 2019;29:540-8.

8. Prete A, Lo AS, Sadow PM, Bhasin SS, Antonello ZA, Vodopivec DM, et al. Pericytes elicit resistance to vemurafenib and sorafenib therapy in thyroid carcinoma via the TSP-1/TGFbeta1 axis. Clin Cancer Res. 2018;24:6078-97.

9. Tsumagari $K$, Abd Elmageed $Z Y$, Sholl $A B$, Green EA, Sobti S, Khan AR, et al. Bortezomib sensitizes thyroid cancer to BRAF inhibitor in vitro and in vivo. Endocr Relat Cancer. 2018;25:99-109.

10. Frazao A, Rethacker L, Jeudy G, Colombo M, Pasmant E, Avril MF, et al. BRAF inhibitor resistance of melanoma cells triggers increased susceptibility to natural killer cell-mediated lysis. J Immunother Cancer. 2020;8:e000275.

11. Ma J, Wang H, Guo S, Yi X, Zhao T, Liu Y, et al. A20 promotes melanoma progression via the activation of Akt pathway. Cell Death Dis. 2020;11:794.

12. Lee B, Sahoo A, Sawada J, Marchica J, Sahoo S, Layng F, et al. MicroRNA-211 modulates the DUSP6-ERK5 signaling axis to promote BRAF(V600E)-driven melanoma growth in vivo and BRAF/MEK inhibitor resistance. J Invest Dermatol. 2020;141:385-94.

13. Valencia-Sama I, Ladumor Y, Kee L, Adderley T, Christopher G, Robinson CM, et al. NRAS status determines sensitivity to SHP2 inhibitor combination therapies targeting the RAS-MAPK pathway in neuroblastoma. Cancer Res. 2020;80:3413-23.

14. Serasinghe MN, Missert DJ, Asciolla JJ, Podgrabinska S, Wieder SY, Izadmehr S, et al. Anti-apoptotic BCL-2 proteins govern cellular outcome following B-RAF (V600E) inhibition and can be targeted to reduce resistance. Oncogene. 2015;34:857-67.

15. Malfatti MC, Gerratana L, Dalla E, Isola M, Damante G, Di Loreto C, et al. APE1 and NPM1 protect cancer cells from platinum compounds cytotoxicity and their expression pattern has a prognostic value in TNBC. J Exp Clin Cancer Res. 2019;38:309.

16. Yuan X, Ma R, Yang S, Jiang L, Wang Z, Zhu Z, et al. miR-520g and miR-520h overcome bortezomib resistance in multiple myeloma via suppressing APE1. Cell Cycle. 2019;18:1660-9.

17. Thakur S, Sarkar B, Cholia RP, Gautam N, Dhiman M, Mantha AK. APE1/Ref-1 as an emerging therapeutic target for various human diseases: phytochemical modulation of its functions. Exp Mol Med. 2014;46:e106.

18. Kelley MR, Georgiadis MM, Fishel ML. APE1/Ref-1 role in redox signaling: translational applications of targeting the redox function of the DNA repair/redox protein APE1/Ref-1. Curr Mol Pharmacol. 2012;5:36-53.

19. Lu GS, Li M, Xu CX, Wang D. APE1 stimulates EGFR-TKI resistance by activating Akt signaling through a redox-dependent mechanism in lung adenocarcinoma. Cell Death Dis. 2018;9:1111.

20. Salzano AM, Paron I, Pines A, Bachi A, Talamo F, Bivi N, et al. Differential proteomic analysis of nuclear extracts from thyroid cell lines. J Chromatogr B Anal Technol Biomed Life Sci. 2006;833:41-50.

21. Liu F, Fu Y, Meyskens FL Jr. MiTF regulates cellular response to reactive oxygen species through transcriptional regulation of APE-1/Ref-1. J Invest Dermatol. 2009;129:422-31.

22. Liu L, Yue Q, Ma J, Liu Y, Zhao T, Guo W, et al. POU4F1 promotes the resistance of melanoma to BRAF inhibitors through MEK/ERK pathway activation and MITF upregulation. Cell Death Dis. 2020;11:451.

23. Wang W, Kang H, Zhao Y, Min I, Wyrwas B, Moore M, et al. Targeting autophagy sensitizes BRAF-mutant thyroid cancer to vemurafenib. J Clin Endocrinol Metab. 2017;102:634-43.

24. Mulcahy Levy JM, Zahedi S, Griesinger AM, Morin A, Davies KD, Aisner DL, et al. Autophagy inhibition overcomes multiple mechanisms of resistance to BRAF inhibition in brain tumors. Elife. 2017;6:e19671.

25. Martin S, Dudek-Peric AM, Maes H, Garg AD, Gabrysiak M, Demirsoy S, et al. Concurrent MEK and autophagy inhibition is required to restore cell death associated danger-signalling in Vemurafenib-resistant melanoma cells. Biochem Pharmacol. 2015;93:290-304.

26. Goodall ML, Wang T, Martin KR, Kortus MG, Kauffman AL, Trent JM, et al. Development of potent autophagy inhibitors that sensitize oncogenic BRAF V600E mutant melanoma tumor cells to vemurafenib. Autophagy. 2014;10:1120-36.

27. Booth LA, Roberts JL, Dent P. The role of cell signaling in the crosstalk between autophagy and apoptosis in the regulation of tumor cell survival in response to sorafenib and neratinib. Semin Cancer Biol. 2020;66:129-39.

28. Garcia-Prat L, Martinez-Vicente M, Perdiguero E, Ortet L, Rodriguez-Ubreva J, Rebollo $E$, et al. Autophagy maintains stemness by preventing senescence. Nature. 2016;529:37-42.
29. Vijayakumar K, Cho GW. Autophagy: an evolutionarily conserved process in the maintenance of stem cells and aging. Cell Biochem Funct. 2019;37:452-8.

30. TCGAR Network. Integrated genomic characterization of papillary thyroid carcinoma. Cell. 2014;159:676-90.

31. Wan PT, Garnett MJ, Roe SM, Lee S, Niculescu-Duvaz D, Good VM, et al. Mechanism of activation of the RAF-ERK signaling pathway by oncogenic mutations of B-RAF. Cell. 2004;116:855-67.

32. Yao Z, Torres NM, Tao A, Gao Y, Luo L, Li Q, et al. BRAF mutants evade ERKdependent feedback by different mechanisms that determine their sensitivity to pharmacologic inhibition. Cancer Cell. 2015;28:370-83.

33. Wee EJ, Wang Y, Tsao SC, Trau M. Simple, sensitive and accurate multiplex detection of clinically important melanoma DNA mutations in circulating tumour DNA with SERS nanotags. Theranostics. 2016;6:1506-13.

34. Gandolfi G, Sancisi V, Piana S, Ciarrocchi A. Time to re-consider the meaning of BRAF V600E mutation in papillary thyroid carcinoma. Int J Cancer. 2015;137:1001-11.

35. Weyemi U, Caillou B, Talbot M, Ameziane-El-Hassani R, Lacroix L, LagentChevallier $\mathrm{O}$, et al. Intracellular expression of reactive oxygen species-generating NADPH oxidase NOX4 in normal and cancer thyroid tissues. Endocr Relat Cancer. 2010;17:27-37.

36. Metere A, Frezzotti F, Graves CE, Vergine $M$, De Luca A, Pietraforte $D$, et al. A possible role for selenoprotein glutathione peroxidase (GPX1) and thioredoxin reductases (TrxR1) in thyroid cancer: our experience in thyroid surgery. Cancer Cell Int. 2018;18:7.

37. De Deken X, Wang D, Many MC, Costagliola S, Libert F, Vassart G, et al. Cloning of two human thyroid CDNAs encoding new members of the NADPH oxidase family. J Biol Chem. 2000;275:23227-33.

38. Guerreiro PS, Corvacho E, Costa JG, Saraiva N, Fernandes AS, Castro M, et al. The APE1 redox inhibitor E3330 reduces collective cell migration of human breast cancer cells and decreases chemoinvasion and colony formation when combined with docetaxel. Chem Biol Drug Des. 2017;90:561-71.

39. Zou GM, Maitra A. Small-molecule inhibitor of the AP endonuclease 1/REF-1 E3330 inhibits pancreatic cancer cell growth and migration. Mol Cancer Ther 2008;7:2012-21.

40. Manguinhas R, Fernandes AS, Costa JG, Saraiva N, Camões SP, Gil N, et al. Impact of the APE1 redox function inhibitor E3330 in non-small cell lung cancer cells exposed to cisplatin: increased cytotoxicity and impairment of cell migration and invasion. Antioxidants. 2020;9:550.

41. Levy JM, Thompson JC, Griesinger AM, Amani V, Donson AM, Birks DK, et al. Autophagy inhibition improves chemosensitivity in BRAF(V600E) brain tumors. Cancer Discov. 2014;4:773-80.

42. Li YJ, Lei YH, Yao N, Wang CR, Hu N, Ye WC, et al. Autophagy and multidrug resistance in cancer. Chin J Cancer. 2017;36:52.

43. Chen M, Hu Y, Hou Y, Li M, Chen M, Tan L, et al. Osteogenesis regulation of mesenchymal stem cells via autophagy induced by silica-titanium composite surfaces with different mechanical moduli. J Mater Chem B. 2020;8:9314-24.

44. Meng X, Wang H, Zhao J, Hu L, Zhi J, Wei S, et al. Apatinib inhibits cell proliferation and induces autophagy in human papillary thyroid carcinoma via the $\mathrm{PI} 3 \mathrm{~K} / \mathrm{Akt} / \mathrm{mTOR}$ signaling pathway. Front Oncol. 2020;10:217.

45. Sueda T, Sakai D, Kawamoto K, Konno M, Nishida N, Koseki J, et al. BRAF V600E inhibition stimulates AMP-activated protein kinase-mediated autophagy in colorectal cancer cells. Sci Rep. 2016;6:18949.

46. Inoki K, Kim J, Guan KL. AMPK and mTOR in cellular energy homeostasis and drug targets. Annu Rev Pharmacol Toxicol. 2012;52:381-400.

47. Mihaylova MM, Shaw RJ. The AMPK signalling pathway coordinates cell growth, autophagy and metabolism. Nat Cell Biol. 2011;13:1016-23.

48. Wu X, Liu Y, Zhang E, Chen J, Huang X, Yan $\mathrm{H}$, et al. Dihydroartemisinin modulates apoptosis and autophagy in multiple myeloma through the P38/MAPK and Wnt/ $\beta$-Catenin signaling pathways. Oxid Med Cell Longev. 2020;2020:6096391.

49. Xu Y, Sun Q, Yuan F, Dong $H$, Zhang $H$, Geng R, et al. RND2 attenuates apoptosis and autophagy in glioblastoma cells by targeting the p38 MAPK signalling pathway. J Exp Clin Cancer Res. 2020;39:174.

50. Robin M, Issa AR, Santos CC, Napoletano F, Petitgas C, Chatelain G, et al. Drosophila p53 integrates the antagonism between autophagy and apoptosis in response to stress. Autophagy. 2019;15:771-84.

51. White E. Autophagy and p53. Cold Spring Harb Perspect Med. 2016;6:a026120.

52. Niehr F, von Euw E, Attar N, Guo D, Matsunaga D, Sazegar $H$, et al. Combination therapy with vemurafenib (PLX4032/RG7204) and metformin in melanoma cell lines with distinct driver mutations. J Transl Med. 2011;9:76.

53. Song $Q$, Liu $H$, Zhen $H$, Zhao $B$. Autophagy and its role in regeneration and remodeling within invertebrate. Cell Biosci. 2020;10:111.

54. Levine B, Kroemer G. Autophagy in the pathogenesis of disease. Cell. 2008;132:27-42.

55. Zhou Y, Wang $Y$, Wu S, Yan Y, Hu Y, Zheng Z, et al. Sulforaphane-cysteine inhibited migration and invasion via enhancing mitophagosome fusion to lysosome in human glioblastoma cells. Cell Death Dis. 2020;11:819. 
14

56. Galluzzi L, Green DR. Autophagy-independent functions of the autophagy machinery. Cell. 2019;177:1682-99.

57. Zheng T, Zhao C, Zhao B, Liu H, Wang S, Wang L, et al. Impairment of the autophagy-lysosomal pathway and activation of pyroptosis in macular corneal dystrophy. Cell Death Discov. 2020;6:85.

58. Yuan P, Ito K, Perez-Lorenzo R, Del Guzzo C, Lee JH, Shen CH, et al. Phenformin enhances the therapeutic benefit of BRAF(V600E) inhibition in melanoma. Proc Natl Acad Sci USA. 2013;110:18226-31.

\section{AUTHOR CONTRIBUTIONS}

$\mathrm{HL}$ wrote the manuscript and planned and performed experiments, data analysis, and figure development; $\mathrm{ZJ}$ and TM completed part of the experiment and obtain the original data; $\mathrm{KN}, \mathrm{XG}, \mathrm{ZJ}, \mathrm{RX}$, and $\mathrm{HX}$ participated in the collection of clinical data; ZW, $\mathrm{YJ}$ and $\mathrm{CL}$ assisted with the experiment; $\mathrm{MW}$ and $\Pi$ provided critical database data analysis for the studies and the manuscript; GM, WX and ZX are responsible for providing funds and reviewing manuscripts.

\section{FUNDING}

This work was supported by grants from the National Natural Science Foundation of China (81872169, 82172821, 82103386, 81702629), Tianjin Municipal Science and Technology Project (19JCYBJC27400), Beijing-Tianjin-Hebei Basic Research Cooperation Project (20JCZXJC00120), and Tianjin Research Innovation Project for Postgraduate Students (2020YJSS178).

\section{COMPETING INTERESTS}

The authors declare no competing interests.

\section{ETHICS APPROVAL AND CONSENT TO PARTICIPATE}

Human experiments were conducted in accordance with the principles of the Declaration of Helsinki, and all patients had signed informed consent. The human experiments and animal studies involved in this study were approved by the Ethics Committee of the Tianjin Medical University Cancer Hospital.

\section{ADDITIONAL INFORMATION}

Supplementary information The online version contains supplementary material available at https://doi.org/10.1038/s41419-022-04550-0.

Correspondence and requests for materials should be addressed to Xiangqian Zheng, Xi Wei or Ming Gao.

Reprints and permission information is available at http://www.nature.com/ reprints

Publisher's note Springer Nature remains neutral with regard to jurisdictional claims in published maps and institutional affiliations.

(c) Open Access This article is licensed under a Creative Commons adaptation, distribution and reproduction in any medium or format, as long as you give appropriate credit to the original author(s) and the source, provide a link to the Creative Commons license, and indicate if changes were made. The images or other third party material in this article are included in the article's Creative Commons license, unless indicated otherwise in a credit line to the material. If material is not included in the article's Creative Commons license and your intended use is not permitted by statutory regulation or exceeds the permitted use, you will need to obtain permission directly from the copyright holder. To view a copy of this license, visit http://creativecommons. org/licenses/by/4.0/.

(c) The Author(s) 2022 